\title{
La répartition des droits de vote, leur exercice et l'efficacité économique
}

\author{
Jacques Hamon ${ }^{1}$ \\ CEREG \\ Université Paris-Dauphine \\ Novembre 2000, dernière version février 2001.
}

\footnotetext{
${ }^{1}$ L'auteur remercie les membres du Conseil Scientifique de la Commission des Opérations de Bourse pour leurs suggestions et commentaires. Les vues exprimées dans ce texte n'engagent que l'auteur qui est bien évidemment seul responsable des erreurs éventuelles. Les exemples cités dans cette étude le sont àtitre d'illustration. Ils ne se veulent pas exhaustifs et peuvent ne pas refléter des changements plus récents.
} 


\title{
La répartition des droits de vote, leur exercice et l'efficacité économique Résumé
}

Cet article présente une revue de la littérature sur la répartition des droits de vote et la défense des intérêts des actionnaires minoritaires. La répartition des droits de vote au prorata de la participation au capital assure généralement la plus grande efficacité économique. La dispersion de l'actionnariat est un cas d'école : en Europe continentale, et notamment en France et en Italie, le capital des entreprises cotées est très concentré et l'actionnariat de type familial est dominant. Un des déterminants le plus significatif de la structure de l'actionnariat est la qualité de la protection juridique accordée aux actionnaires minoritaires. Les marchés les plus développés relativement àl'importance de l'économie sont également ceux où les droits des actionnaires minoritaires sont les mieux protégés par la loi ou la réglementation.

\section{Ownership concentration, corporate voting rights and the efficiency of capital allocation}

\begin{abstract}
This paper surveys research on corporate governance, with special attention to the importance of legal protection of investors and of ownership concentration in corporate governance systems in Europe. One share-one vote encourages the selection of an efficient management team. Families are the most pronounced type of controlling shareholders in Europe, especially in France and Italy. Investor protection (by law) turns out to be crucial because, in many countries, expropriation of minority shareholders by the controlling shareholders is extensive. The efficiency of capital allocation is positively correlated with the legal protection of investors. The agency model predicts larger capital markets in countries where agency costs are reined in by the law and the institutions built to support their enforcement.
\end{abstract}

JEL : G32, K22, D23, G34, L21

Keywords: investor protection, corporate governance, ownership concentration, one share-one vote, law 


\section{Sommaire}

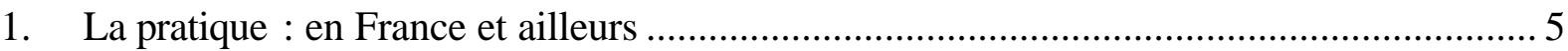

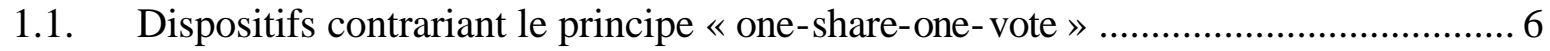

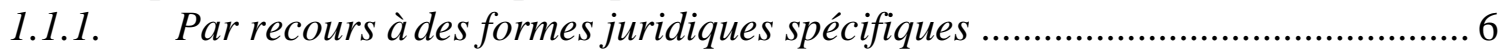

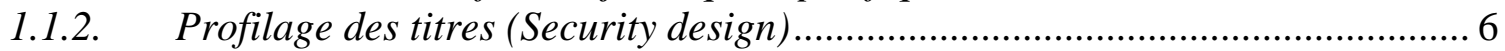

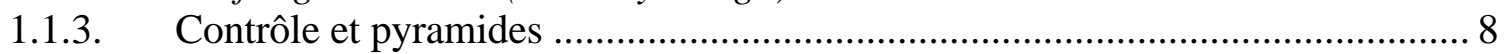

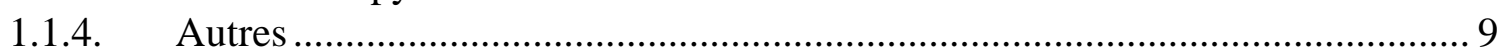

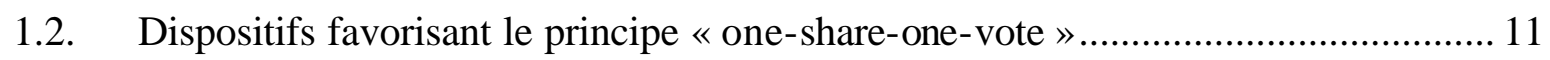

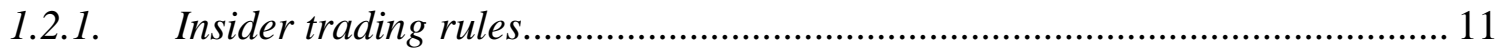

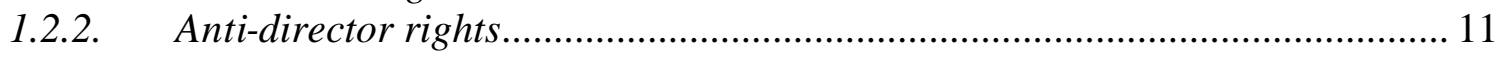

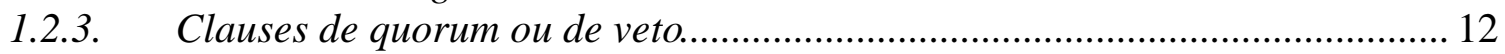

1.2.4. Dynamisation et contrôle des investisseurs institutionnels ............................ 12

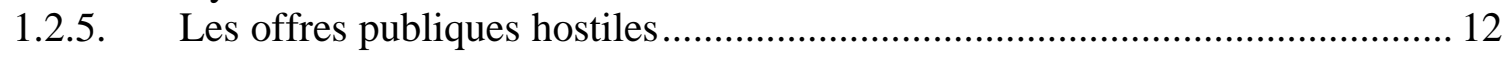

2. La théorie : structure de l'actionnariat et efficacité économique ................................... 12

2.1. L'hypothèse d'une privatisation du flux de bénéfices .......................................... 13

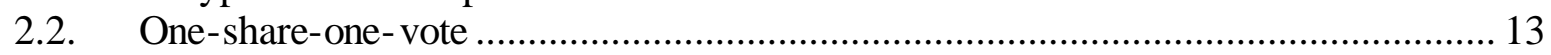

2.3. Surveillance et structure de l'actionnariat ........................................................... 15

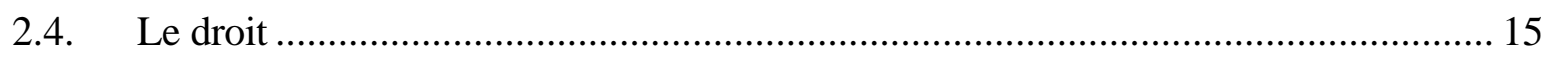

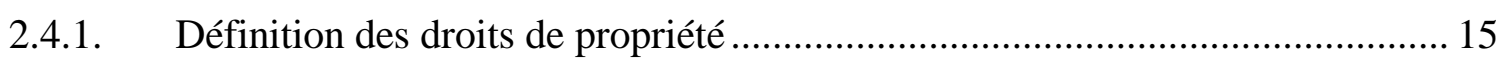

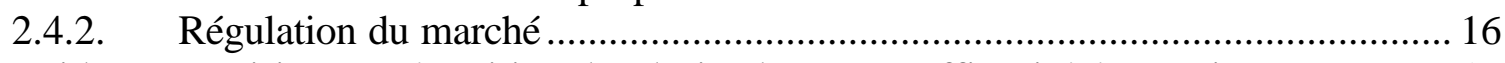

3. Evidence empirique : répartition des droits de vote et efficacité économique ................. 16

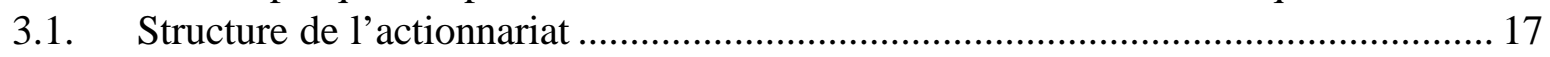

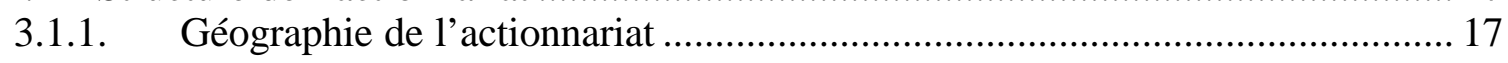

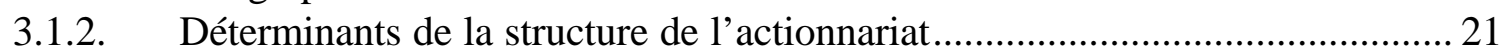

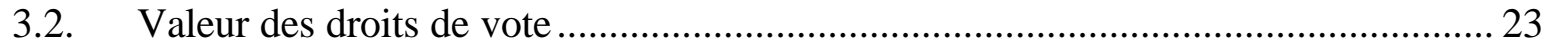

3.2.1. Différence de valeur entre deux catégories d'actions à droits de vote

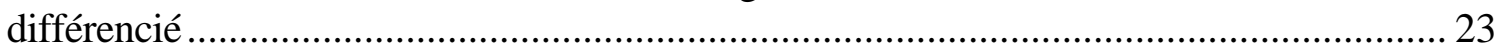

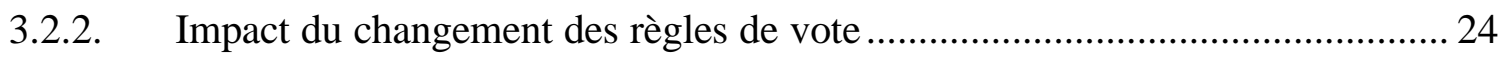

3.2.3. L'importance des bénéfices privés .............................................................. 24

3.3. Structure de l'actionnariat et efficacité économique ........................................... 24

3.3.1. Développement des marchés et contribution au financement des entreprises. 25

3.3.2. Diffusion d'informations et protection des minoritaires................................ 25

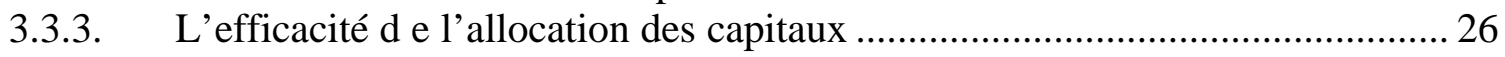

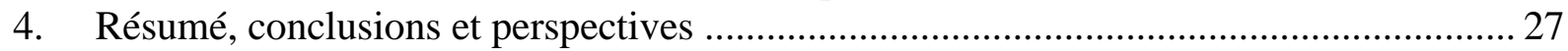

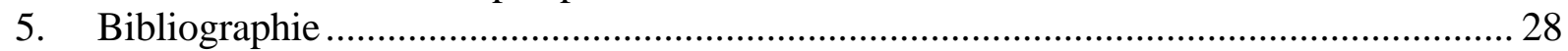




\section{La répartition des droits de vote, leur exercice et l'efficacité économique}

Dans un monde sans frictions la répartition des flux résiduels générés par une entreprise est strictement proportionnelle aux participations aux capitaux propres des différents actionnaires et le problème du pouvoir et de son exercice ne se pose pas.

La question du pouvoir apparaît lorsque le contrôle permet de répartir différemment les flux entre les propriétaires/actionnaires, lorsque les intérêts des dirigeants et des actionnaires exerçant le contrôle (insiders) ne coï ncident pas ou plus avec ceux des autres actionnaires (outside investors). C'est dans cette situation de conflits potentiels que la question de la répartition et de l'exercice des droits de vote devient cruciale.

Les actionnaires notamment minoritaires ou ne participant pas aux conseils d'administration demanderont une répartition des droits de vote au prorata de la participation au capital, au nom de la transparence, de l'équité, et de façon à éviter qu'une partie des ressources de l'entreprise ne soit accaparée par une minorité active. Les entrepreneurs souhaiteront pouvoir mettre en ouvre leurs projets et pour cela lever les fonds nécessaire sans perdre le contrôle de leur société.

Shleifer et Vishny (1997) soutiennent que la séparation entre la gestion de l'entreprise et sa propriété entraîne un problème d'agence et un conflit entre les dirigeants et un actionnariat dispersé aux Etats-Unis, alors que ce conflit intervient ailleurs entre actionnaires minoritaires et actionnaires exerçant le contrôle.

Plusieurs questions sont posées sur les plans institutionnels, théoriques et empiriques :

- Sur le plan théorique : quels sont les avantages et inconvénients du principe «oneshare-one-vote $\gg ?$

- Sur le plan des institutions, de la définition des titres et du droit : quels sont les divers dispositifs permettant d'éviter ou de contourner le principe «one-vote-one-share »? Ces mécanismes sont-ils les mêmes en France et dans les autres pays? Quelle est l'importance et la place de la régulation, de la définition de contraintes légales s'opposant la libre rédaction des contrats entre les différents apporteurs de capitaux et les dirigeants des entreprises?

- Sur le plan empirique : quelle est la structure de l'actionnariat en France, se distinguet-elle de la situation des autres pays européens? et de celle des entreprises EtatsUniennes? Quels sont les actionnaires ultimes? Quelle est la concentration du pouvoir?

- Et bien sûr, les pratiques discriminantes de votes ont-elles une efficacité économique? Créent-elles de la valeur et pour qui? Ou bien ne sont-elles pas au contraire un ensemble de dispositifs permettant aux dirigeants ou àun groupe d'actionnaire d'échapper à la sanction du marché et ne favorisent-elles pas l'enracinement des dirigeants?

Ce texte propose une revue de la littérature traitant de ces questions, il comporte trois sections : dans une première on recense les pratiques existantes en détaillant le cas français ; la deuxième section présente certains développements théoriques et les arguments en faveur d'une modalité particulière de répartition des droits de vote; la troisième section détaille les études empiriques sur la géographie de l'actionnariat, la valeur des droits de vote, l'incidence d'une défense des intérêts des actionnaires minoritaires sur la valeur de l'entreprise et la croissance. 


\section{La pratique : en France et ailleurs}

La séparation entre le droit aux flux futurs et le droit de vote répond àdivers objectifs : particuliers évidemment, puisque sous l'hypothèse de comportement habituellement spécifiée en théorie financière il est plus agréable de contrôler une société sans y apporter de capitaux ou en y mettant le moins de capitaux possibles. Le contrôle permet d'envisager dans ce cas des décisions qui ne seront plus forcément prises dans l'intérêt de tous les actionnaires, mais qui avantageront la minorité aux affaires. La privatisation d'une partie du flux bénéficiaire peut être réalisée de différentes manières : en surinvestissement dans des projets ne maximisant pas la valeur de marché des actions (pet project), en surconsommation au profit d'une minorité, en affaires conclues avec des entreprises qui elles, sont la propriété exclusive de la minorité contrôlant l'entreprise. Et plus généralement en suivant toute politique qui ne maximise pas la valeur de l'ensemble des actions. Le courant sur la « création de valeur pour l'actionnaire » (shareholder value) a repris cette proposition classique suivant laquelle un projet d'investissement n'est créateur de valeur que dans la mesure où le taux de rentabilité attendu est supérieur au coût du capital ${ }^{2}$.

D'autres arguments apparemment moins égoï stes ont été également avancés par exemple lorsque l'actionnaire exerçant le contrôle est l'Etat. Ce qui justifie l'exercice d'un droit de veto sur des opérations contraires à l'intérêt national (par le biais d'une « golden share »), et la possibilité de peser sur les décisions de l'entreprise dans le sens de l'intérêt général. Toutefois ce dernier argument est plus facilement compréhensible lorsque l'Etat est le seul propriétaire. La multiplication d'affaires récentes ont montré que l'intérêt général pouvait n'être que l'arbre cachant la forêt des intérêts particuliers ${ }^{3}$.

A certaines époques on a également pu opposer l'intérêt de l'entreprise et celui de ses actionnaires. Les grandes sociétés hollandaises ${ }^{4}$ verrouillent leur capital, et s'appuient sur des possibilités accordées par le législateur et rarement rencontrées ailleurs. Certains rappellent la Compagnie des Indes Orientales, une des premières sociétés par actions, qui affichait statutairement que "le bien de l'entreprise devait primer sur celui des actionnaires". La menée des affaires avec plusieurs bateaux affrétés pour des expéditions risquées, exigeait une « vision de long terme » que les actionnaires avec des intérêts « purement financiers » et de qui plus est une vision de court terme ne pouvaient pas avoir. Le développement des télécommunications et des moyens d'informations n'a pas empêché le maintien des dispositifs législatifs hollandais qui attirent de plus en plus de sociétés européennes ${ }^{5}$ à l'image des localisations dans le Delaware aux Etats-Unis!

2 Le coût du capital, dans la théorie financière est la rentabilité exigée par les actionnaires sous l'hypothèse d'un financement exclusif par capitaux propres, du moins si on admet la neutralité du choix d'une structure de financement selon l'argument d'arbitrage de Modigliani et Miller (1958).

3 Comment justifier les 150 milliards de francs perdus par le Crédit Lyonnais ? les 50 milliards (ou plus ?) perdus par le Gan? La restructuration de l'UAP n'a-t-elle pas été étalée dans le temps pour ne pas gêner la BNP? La constitution des « noyaux durs » a-t-elle toujours été faite en pensant aux intérêts de tous les actionnaires, par exemple Saint Gobain aurait-il pris les mêmes participations au capital des privatisées successives? Cet argent distrait dans les noyaux durs ne pouvait-il pas avoir d'autres utilisations? Sans parler des « affaires » où la rémunération de personnes dont l'efficacité pour l'entreprise n'apparaît pas évidente...

4 Heineken, ABN Amro, Phillips, ING... En Hollande il est possible d'émettre des actions prioritaires au profit d'une fondation contrôlée par les dirigeants et qui a le droit exclusif de nommer les dirigeants, et de changer les statuts. Si plus de 100 employés et un capital et réserves de plus de 25 millions de florins il est possible de mettre en place un conseil de surveillance dont les membres sont élus par cooptation et qui nomme les membres de la direction. Toujours en Hollande, il est possible d'augmenter le capital sans en référer aux actionnaires existants.

5 Voir les rebondissements de l'affaire Gucci (avec B. Arnault dans le rôle du minoritaire opprimé). 
La séparation entre le droit aux flux futurs et le droit de vote est obtenue de différentes manières.

\subsection{Dispositifs contrariant le principe « one-share-one-vote »}

\subsubsection{Par recours à des formes juridiques spécifiques}

La société en commandite par actions organise la séparation entre la gestion (assurée par le ou les commandités) et l'apport de fonds propres (assuré par les commanditaires). Les commandités étant responsables sans limitation sur leurs biens personnels, alors que les commanditaires sont responsables à concurrence de leurs apports. C'est le dispositif idéal anti-OPA ou permettant la transmission filiale de la direction de l'entreprise, tout en n'apportant qu'une faible partie des capitaux propres.

Cette forme juridique est particulièrement étonnante pour une société cotée en bourse et semble une spécificité française non récente d'ailleurs (Michelin). L'étonnement est encore plus grand lorsque le commandité est une société à responsabilité limité (Eurodisney, Office Commercial Pharmaceutique).

\subsubsection{Profilage des titres (Security design)}

\subsubsection{A l'aide de titres spécifiques} différents des actions ordinaires

Plusieurs catégories de titres sont émis et font l'objet de cotation séparée.

Emission d'actions sans droit de vote (non-voting stock). C'est une pratique que l'on retrouve dans toutes les législations : ainsi en France depuis 1978, les actions à dividendes prioritaires sans droits de vote (ADP). Généralement la proportion de ce type de titres dans les capitaux propres des entreprises est contrainte et le revenu versé aux porteurs de titres sans droits de vote peut être supérieur à celui des actionnaires pour une mise de fonds identique. Ainsi dans le cas français, les ADP sont limitées à $25 \%$ du capital et une omission du dividende amène les porteurs d'ADP à récupérer un droit de vote sous certaines conditions. Les ADP sont des titres spécifiques faisant l'objet d'une cotation séparée.

Emission de plusieurs classes d'actions avec droits de vote différenciés ou plural, ce qui constitue l'extension du cas précédent : une catégorie A associera un droit de vote à chaque action, une catégorie B en associera 2 ou plus. Bien sûr les titres sont différents, s'ils sont cotés en bourse ils le sont sur des lignes de cotation différentes et s'échangent ainsi avec des valeurs différentes. Ce type de titres est rencontré dans la plupart des pays européens, le cas limite étant celui de la « golden-share ${ }^{6}$.

Jusqu'en 1984 le NYSE a systématiquement radié les entreprises (5 en fait) émettant une seconde classe d'actions avec droits de vote multiple. En 1984, le NYSE a changé de politique sous la pression de General Motors qui souhaitait émettre une seconde classe d'actions pour financer une acquisition. Une première période (le moratoire) a été suivie à partir de septembre 1986 d'une autorisation àémettre des classes d'actions nouvelles avec droit de vote différent. A l'époque, l'AMEX comme le NASDAQ admettaient ce type d'émission sans restriction pour le NASDAQ et à la condition d'un ratio de droits de vote n'excédant pas 1 pour 10 dans le cas de l'AMEX. La règle 19-c-4 de la SEC admet l'émission de nouveaux titres à condition que les droits de vote des titres existants ne soient pas réduits,

6 En Suisse on remarque une grande diversité de types d'actions avec des droits notamment de vote différents, ou la possibilité de restreindre la détention de certains titres à des nationaux. Ces actions sont le plus souvent cotées séparément. Ces divers mécanismes rendent difficile une éventuelle prise de contrôle de société (voir Loderer et Jacobs, 1995). 
l'admission d'actions nouvelles sans droit de vote est donc autorisée. Pour une société déjà cotée, la création d'une catégorie d'actions peut-être réalisée de trois façons dénommées par Jarrell et Poulsen (1988), la méthode dividende, la méthode échange et la méthode durée :

- La méthode « dividende » consiste en une distribution d'actions gratuites sans droits de vote; ou une distribution d'action avec un droit, les actions existant avant s'en voyant dans ce dernier cas attribuer dix par exemple. Quelquefois la classe à faible droit n'a pas de droits de vote mais un quota de représentation au conseil d'administration en échange. Il y aura ainsi non plus une, mais deux lignes de cotation en bourse. A l'issue de cette première étape la répartition du pouvoir entre les anciens actionnaires n'est pas modifiée. Quant aux nouveaux actionnaires, c'est en connaissance de cause qu'il achèteront soit l'action àfaible ou bien celle à fort droit de vote.

Le plus souvent les actionnaires anciens sont cependant incités à échanger une pour une leurs actions anciennes contre des nouvelles à faible droit de vote et le plus souvent ils l'acceptent, en raison d'un super-dividende (10\% de plus que le dividende ordinaire le plus souvent) versé aux actions nouvelles dont les droits de vote sont inférieurs. Les insiders, conservent les actions à fort droit de vote et souvent renforcent leur pouvoir de vote àl'occasion de cette opération.

- La méthode échange consiste à créer des actions nouvelles à droit de vote renforcé, les actions anciennes devenant donc la classe à faible droit de vote. Un dividende amélioré peut être servi aux actions àfaible droit. Dans une deuxième étape un échange est proposé (self tender offer) et la négociabilité des actions à fort droit de vote peut être contrainte. Ceux qui souscrivent àl'échange appartiennent évidemment plutôt au groupe des insiders.

- La méthode durée consiste dans une première étape à attribuer par exemple dix droits de vote à chaque action existante, puis à créer une deuxième classe d'actions auxquelles seul un droit de vote est associé. Le passage d'une classe à l'autre est conditionné par une durée de détention. Après la création des deux classes, tout achat d'actions ne peut concerner qu'une action courte, qui devient longue après une période de détention généralement de 4 ans.

L'exercice du droit de vote pour l'élection des membres du conseil d'administration peut aux Etats-Unis se faire dans un collège commun avec des quotas de représentation ou bien dans des collèges séparés (Ruback, 1988, p 156). Le système des collèges séparés permet de garder le contrôle avec encore moins de capitaux, si les quotas sont judicieusement fixés.

Certains pays ont autorisé des « parts bénéficiaires » auxquelles sont associés des droits de vote, ces parts peuvent être attribuées sans apports en capital (en Belgique actuellement). Les actions-reflet appelées «tracking stock » ou «targeted stock» aux Etats-Unis (autorisée dans l'Etat du Delaware) consistent à mettre sur le marché une ou plusieurs catégories d'actions liées à une branche d'activité de l'entreprise, branche non forcément filialisée ${ }^{7}$. Il s'agit d'un type de titres facilitant la dissociation entre les droits financiers et les droits de vote sauf à contrôler très rigoureusement les transferts de personnels, d'actifs, ou les changements de périmètres. L'avantage pour la société émettrice (ou ses dirigeants) est

\footnotetext{
${ }^{7}$ La première opération française est proposée par Alcatel en octobre 2000 pour sa division Optronics, non filialisée (en fait la division comporte une filiale française et une autre implantée aux Etats-Unis). Alcaltel-O est cotée à Paris et sous forme d'ADR au NASDAQ, parallèlement à Alcatel-A « action ordinaire » également cotée sur diverses places dont Paris. Suivant le rapport du groupe de travail de la Commission des Opérations de Bourse présidé par M. Barbier de la Serres, mars 2000 : «Toutefois, si elle est souhaitable, la filialisation ne constitue pas un préalable nécessaire àl'émission d'actions traçantes. L'émetteur peut, en effet, considérer, pour des raisons d'organisation interne, qu'il est préférable que l'activité suivie soit répartie entre plusieurs de ses filiales ou constituer un département de la société-mère ou de l'une de ses filiales».
} 
d'éviter toute prise de contrôle extérieure sur la division faisant l'objet de l'émission reflet. Le calcul de la valeur de l'action reflet suppose notamment une évaluation des droits de l'actionnaire-reflet en cas de disparition ${ }^{8}$ ce qui ne semble pas évident.

\subsubsection{A l'aide de titres spécifiques non indépendants des actions ordinaires}

Les certificats d'investissement sont des actions démembrées : l'action est éclatée en un certificat d'investissement et un certificat de vote. Les certificats de vote sont distribués aux anciens actionnaires au prorata des droits de vote détenus. Les porteurs de certificats d'investissement ont les mêmes droits que les actionnaires, notamment le droit au dividende, sauf le droit de vote. Les droits de vote sont négociables mais il est nécessaire d'être en possession d'un certificat pour avoir le droit d'acheter un droit de vote.

L'aspect extrêmement bizarre de cet instrument est la création de fait de deux catégories d'actionnaires : ceux qui étaient actionnaires avant l'émission des certificats et ceux qui le sont après. Les premiers auront reçu, et souvent gratuitement notamment entre 1983 et 1988 lorsque les droits de vote n'étaient pas négociables, des droits de vote supplémentaires. Ce type de titres a été créé en 1983 par l'Etat français qui voulait renforcer les fonds propres des entreprises publiques tout en refusant la privatisation du capital.

\subsubsection{A l'aide de clauses statutaires s'appliquant à des titres apparemment classiques}

Une spécificité française est la possibilité de droit de vote double donné par la loi de $1966^{9}$. Ces droits de vote double, qui nécessitent une disposition statutaire, sont accordés aux actionnaires de l'entreprise sous condition d'une durée de détention des actions. La durée est entre 2 et 4 ans.

Alors qu'en bourse une seule action sera cotée, avec un prix affiché; deux biens co-existeront un premier représentant l'action ordinaire et son droit de vote simple, le second est l'action ordinaire et son droit de vote double. Le droit de vote supplémentaire n'est pas négociable et est perdu en cas de revente de l'action.

L'objectif annoncé est de « fidéliser l'actionnariat », au prix, on le remarque d'une réduction de la liquidité du titre et d'une contrainte en matière de gestion de portefeuille.

\subsubsection{Contrôle et pyramides}

\subsubsection{Par autocontrôle}

L'autocontrôle a beaucoup diminué en France depuis 1991, date à laquelle le droit de vote des actions en auto-contrôle a été supprimé.

\footnotetext{
8 suivant le prospectus d'Alcatel, qui comporte 201 pages « entraînent la transformation des actions de catégorie $O$ en actions ordinaires de catégorie A avec, selon le cas, soit indemnisation par Alcatel des porteurs $d$ 'actions de catégorie $O$ (en actions de catégorie A ou en espèces), soit sans indemnisation. L 'indemnité éventuellement due est fonction du Produit Net par Action de catégorie O résultant ou ressortant de l'opération de cession ou de mise en bourse, tel que défini àl 'article 13.2 des statuts et déterminé à dire d 'expert, et des Moyennes des Cours des actions A et des actions de catégorie O, telles que définies également àl'article 13.2 des statuts », (...) «les détenteurs d'actions de catégorie O seront des actionnaires d 'Alcatel, participant aux risques de l'ensemble de ses activités, sans droit spécifique sur les actifs de la Division Optronics, et sans représentation spécifique au sein du conseil d 'administration d 'Alcatel ou au sein de la Division Optronics » (...) «Les porteurs d'actions de catégorie $O$ ne disposent d'aucun droit spécifique sur les actifs de la Division Optronics ».

9 Interdit en Italie, Espagne et Grande-Bretagne et interdit depuis mai 1998 en Allemagne (Faccio et Lang, 2000, $\mathrm{p} 21)$
} 
Le ramassage en bourse « en vue de régulariser le cours » constitue toutefois une forme de plus en plus utilisée de rachat de ses propres actions par une société, les actions ainsi ramassées n'étant pas forcément annulées.

1.1.3.2. Par un contrôle en cascade et des participations croisées Une cascade de sociétés permet à celui ou au groupe contrôlant le holding de tête de contrôler une société située quelques échelons plus bas tout en n'ayant qu'une faible part du capital de la dite société. Les apports en capitaux sont faits par divers actionnaires tout au long de la chaîne entre le holding de tête et la société.

Même en supposant une parfaite assiduité des actionnaires aux assemblées générales et un vote groupé et hostile à la direction, en contrôlant $50 \%$ des droits de vote on garde la maîtrise de la société cotée. On loge cette participation dans une deuxième société qui elle même est contrôlée à $50 \%$ par une troisième... jusqu'au holding de tête qui par plus de sécurité ne sera pas coté en bourse. Ces constructions pyramidales sont une spécialité européenne (italienne ou française) ou asiatique.

Bien sûr avec des mécanismes de droits de vote double et un pari raisonnable quant au degré d'hostilité des minoritaires ou àleur assiduité aux assemblées, la mise de fonds dans la chaîne de société est encore plus modeste. Dans certains cas « l'investisseur » contrôlant l'ensemble de la pyramide n'aura quasiment rien investi («voting trust » en tête de chaîne ainsi que le droit hollandais le permet). En France ou en Belgique, les banques sont actives dans ces chaînes (voir le rapport ECGN de Becht, $1997^{10}$ ).

Plus la chaîne est longue et plus la distorsion sera grande au principe du «one-share-onevote ». Ces constructions pyramidales sont de plus difficiles à reconstituer et l'identité de l'actionnaire ou du groupe qui détient le contrôle de fait n'est pas forcément évidente à identifier.

Bien sûr les bonnes questions concernent l'efficacité économique des constructions pyramidales? Les fonctions de contrôle (monitoring) sont-elles assurées par les holdings de tête? Quelle est l'importance de l'évaporation des flux financiers vers ces holdings qui quelquefois facturent des services (voir notamment Lagardère en 1994)?

\subsubsection{Autres}

\subsubsection{En plafonnant explicitement \\ l'exercice du droit de vote}

La limite à l'exercice du droit de vote, doit être approuvée par les actionnaires. Ensuite le nombre de droits de vote qu'un actionnaire peut exprimer est plafonné. Il est ensuite nécessaire de contrôler les deux tiers du capital pour retrouver la totalité de ses droits de vote! Selon le quotidien La Tribune du 28 avril 2000, Vivendi aurait fait approuver en avril 2000 une «clause d'ajustement du nombre de droits de votes exprimables en assemblée par tout actionnaire détenant une participation dans le capital supérieure à $2 \%$, clause ne s'appliquant pas au Président de la société : suivant le calcul fait par Déminor, sur la base d'un quorum de $49,9 \%$, un groupe d'actionnaire qui aurait $50,10 \%$ des droits de vote avant, en aurait $29,26 \%$ après et perdrait ainsi la majorité; tandis que dans l'hypothèse où le Président contrôle $32,06 \%$ des droits de vote avant, le dispositif lui permettrait d'obtenir la majorité absolue.

\subsubsection{Par expropriation}

Les actionnaires peuvent renoncer à leur droit préférentiel de souscription, ce qui ensuite les expose à une dilution. Cette décision prise majoritairement s'applique à tous les actionnaires.

\footnotetext{
10 "Hence, banks provide the "glue" for group structures that are used to separate ownership and control."
} 
La loi française du 31 décembre 1993 précise les conditions du retrait obligatoire (squeeze out) à la demande d'un actionnaire détenant plus de $95 \%$ du capital d'une société cotée. La vente forcée sera conclue à un prix fixé par un expert indépendant, proposé par l'entreprise, agréé par le CMF, avec droit de veto de la COB.

Il s'agit d'une exception à l'article 545 du code civil selon lequel 'Nul ne peut être contraint de céder sa propriété, si ce n'est pour cause d'utilité publique”. Des opérations de retrait obligatoire ont impliqué de grandes sociétés cotées comme Total, Michelin, Nestlé, Indosuez, BNP, Schlumberger ou Société générale (sur Sogenal).

\subsubsection{Par entente entre plusieurs actionnaires}

Les ententes entre actionnaires peuvent prendre plusieurs formes telles que les pactes d'actionnaires, les noyaux durs ou des contrats optionnels de gré à gré. Les pactes d'actionnaires peuvent comporter des clauses d'harmonisation des votes ou des clauses de préemption en cas de cession de la participation d'un des signataires.

\subsubsection{Dépôt des droits de vote}

L'actionnaire dépose ses titres auprès d'un intermédiaire qui peut dans certains pays avoir le droit d'exercer les droits de vote soit par accord explicite soit même par défaut (cas des Depotstimmrecht allemands).

\subsubsection{Mesures contrariant la liquidité du titre}

Plusieurs dispositifs contrarient la liquidité du titre notamment la réglementation accordant les droits de vote double sous condition de détention ou le ramassage en bourse « en vue de régulariser le cours ». Parmi les dispositifs non encore mentionnés, on note : l'obligation de dépôt des titres avant une assemblée générale, et la pratique d'un dividende renforcé sous condition de durée de détention de l'action ${ }^{11}$. Le dividende majoré accorde par ailleurs une prime aux groupes d'actionnaires exerçant le contrôle de fait et n'ayant évidemment pas l'intention de se séparer de leurs titres.

\subsubsection{Mesures contrariant la transparence}

La mise en nominatif des actions permet de garder la trace en SICOVAM des identités des porteurs successifs de l'action et de retracer la structure de l'actionnariat. Cette connaissance est toutefois limitée notamment dans le cas où l'actionnaire de la société française est lui même étranger : dans ce cas, le plus souvent, dans les livres de la SICOVAM ne figurera que l'identité de l'un des intermédiaires impliqué dans la transaction.

Le dispositif introduit surtout une grande inégalité d'accès à l'information. puisque la structure de l'actionnariat est dans ce cas publique mais facturée ce qui la réserve essentiellement à la direction de l'entreprise.

Certaines mesures favorisent la transparence et améliorent la connaissance de la structure de l'actionnariat. Dans le cas français on note l'obligation de déclaration des franchissements de seuils (à la hausse comme à la baisse, lois du 17 juin 1987, du 2 août 1989) et la publication de ces déclarations. L'absence de déclaration peut entraîner une amende et la perte du droit de vote pour deux ans. Les seuils définis en 1989 sont de 5, 10, 20, 33, 50 et 66\%. Des dispositions statutaires, donc propres aux entreprises, peut compléter le dispositif en introduisant des seuils intermédiaires (LVMH ou Danone ont par exemple fixé une déclaration obligatoire à $1 \%$ ).

11 Air Liquide supprime en 1993 le droit de vote double et "en échange” crée le dividende majoré de 10\%, plusieurs sociétés ont adopté depuis le dividende majoré. 


\subsection{Dispositifs favorisant le principe « one-share-one-vote 》}

Les mécanismes de protection des intérêts des minoritaires, les mécanismes contrariant les stratégies de confiscation des bénéfices au profit d'un groupe d'actionnaires, les mesures renforçant les droits des actionnaires notamment minoritaires face aux dirigeants et/ou actionnaire exerçant le contrôle sont successivement étudiés ci après.

\subsubsection{Insider trading rules}

Aux Etats-Unis un insider est tout actionnaire détenant $10 \%$ ou plus du capital d'une société, de même que tout dirigeant de la même société.

Des réglementations permettent de contrôler toutes les transactions faites par un insider sur les titres de la société. Les déclarations sont obligatoires, recensées par la SEC et publiées avec un léger décalage sur le site de la SEC (Official Summary of insider trading). Ces déclarations sont reprises par la plupart des médias, quel que soit le nombre d'actions achetées ou vendues (voir par exemple www.msn.com).

La réglementation sur la détention et l'utilisation d'information sensible, précise que soit l'insider n'opère pas en bourse sur les titres de la société, soit il diffuse l'information qu'il détient avant la transaction en bourse. Cette épée de Damoclès qui pèse sur tout détenteur de blocs de titres, introduit un coût à la détention de blocs de plus de $10 \%$ et amène de fait à limiter la part détenue par de nombreux actionnaires dans une société : on observe aux EtatsUnis une concentration inhabituelle des participations au capital à un niveau légèrement inférieur au seuil définissant l'insider. En Europe les détenteurs de blocs de titres ne sont pas a priori considérés comme initié.

\subsubsection{Anti-director rights}

Sous le terme « anti-director rights », la littérature anglo-saxonne recense diverses dispositions visant à offrir des protections légales contre les tentatives d'expropriation d'actionnaires minoritaires, par la direction ou les actionnaires majoritaires. Ces dispositions concernent les conditions d'exercice du droit de vote et les possibilités de recours. La Porta et al (1998) proposent un indicateur synthétique, caractérisant un pays donné et obtenu en répondant aux questions suivantes :

- Le vote par procuration est-il possible ou interdit? (possible en France, 1 point)

- Y-a-t-il obligation de dépôt des titres avant le vote? (dépôt obligatoire trois jours avant l'assemblée générale en France, 0 point) ${ }^{12}$

- Le pourcentage de capital nécessaire àl'obtention de l'organisation d'une assemblée générale extraordinaire est-il supérieur à 10\% (égal à 10\% en France, 1 point)

- Le « vote cumulé » ${ }^{13}$ est-il autorisé dans l'élection des membres du conseil d'administration? (pas autorisé en France, 0 point)

- Est-on obligé de consulter les actionnaires avant de supprimer leur droit de souscription préférentiel? (oui en France, 1 point)

- Y-a-t-il obligation de représentation de minorités au conseil d'administration ? Y-a-t-il possibilité de contester certaines décisions devant tribunaux? Y-a-t-il possibilité d'obliger à racheter la part des minoritaires à leur demande? (selon La Porta et al, 0 pour la France)

\footnotetext{
${ }^{12}$ Le dépôt des titres a pour objectif d'identifier les actionnaires effectifs au moment même du vote, son inconvénient est de contrarier les transactions à un moment crucial dans la vie de la société ce qui peut amener certains actionnaires àne pas exercer leurs droits de vote.

13 « cumulative voting », dans l'hypothèse où 5 membres sont à élire, le vote cumulé est autorisé si tout actionnaire a 5 voix, et si il peut regrouper ses 5 voix sur un seul nom.
} 
L'indicateur de La Porta et al a par définition une valeur comprise entre 0 et 6 . La valeur est de 3 pour la France, contre 5 pour les Etats-Unis ou la Grande-Bretagne et 1 pour l'Allemagne et l'Italie. Bien sûr, les questions pourraient être précisées et d'autres indicateurs envisagés.

\subsubsection{Clauses de quorum ou de veto}

Les modifications statutaires doivent être approuvées par une majorité des deux-tiers. A titre d'exemple, selon le quotidien La Tribune du 28 avril 2000, dans le cas du plafonnement des droits de vote de Vivendi, la mesure aurait été approuvée par 73,63\% des votes exprimés avec un quorum de $28,6 \%$.

Certaines modifications de statuts sont quasiment irréversibles, leur adoption par des assemblées où le quorum est très faible apparaît très contestable. Deux propositions ont été faites dans la littérature :

- l'introduction d'une clause de quorum pourrait éviter de voir les statuts modifiés par une minorité;

- on peut également envisager avec Hart (1995, p. 209), l'introduction d'une clause de veto : une proportion donnée (faible) d'actionnaires pouvant s'opposer à toute modification des statuts, notamment en cas de remise en cause des structures de vote.

\subsubsection{Dynamisation et contrôle des investisseurs institutionnels}

Pour différentes raisons, les fonds gérant l'épargne collective investie en actions ont eu, aux Etats-Unis, une attitude plutôt passive n'exerçant pas le plus souvent leurs droits de vote. En 1988, le département du travail a obligé les investisseurs institutionnels (dont les fonds de pension) à l'exercice de leurs droits de vote. En juillet 1994, cette obligation a été étendue à l'étranger. Ce qui a amené les fonds américains à s'organiser et à créer des structures fédératives de plus en plus actives.

\subsubsection{Les offres publiques hostiles}

Le mécanisme des offres publiques hostiles et ses surenchères protège les actionnaires minoritaires qui voient ainsi la valeur de l'entreprise cible être révélée. L'offre publique hostile révèle la valeur de l'entreprise et celle des droits de vote.

Un dépôt d'OPA est obligatoire en France si un actionnaire détient plus d'un tiers du capital ou des droits de vote. De (très) nombreuses dérogations ont toutefois été accordées, dispensant les sociétés ou actionnaires contrôlant plus du tiers de lancer une offre.

\section{La théorie : structure de l'actionnariat et efficacité économique}

L'efficacité économique est appréciée de différentes manières mais toujours du point de vue des actionnaires dans la littérature ici passée en revue ${ }^{14}$. L'optimalité d'une structure de droits de vote peut ainsi être définie comme liée au choix de la «meilleure équipe dirigeante », qui est celle qui obtient la valeur de marché des capitaux propres la plus forte.

\footnotetext{
${ }^{14}$ Le numéro spécial du Journal of Financial Economics de janvier-mars 1988, intitulé « The distribution of power among corporate managers, shareholders, and directors », sous la direction de M. Jensen et J. Warner, contient de nombreuses contributions qui font toujours autorité sur le sujet. On peut aussi se reporter à Hart (1995) ou à Shleifer et Vishny (1997).
} 


\subsection{L'hypothèse d'une privatisation du flux de bénéfices}

Si un groupe d'actionnaires tire du contrôle de l'entreprise des avantages financiers et ainsi obtient un partage des richesses générées par l'entreprise non proportionnel àla participation au capital, on parle de privatisation de bénéfices dans la littérature (private benefits). Le gouvernement d'entreprise définit un ensemble de procédures permettant d'éviter l'expropriation d'actionnaires, le plus souvent minoritaires par les insiders (c'est à dire les dirigeants et/ou le groupe des actionnaires exerçant le contrôle) ${ }^{15}$. L'expropriation, quant elle se produit, peut prendre différentes formes :

- un vol pur et simple dans certains pays;

- une cession d'actif, à un prix sous-évalué, à une entité juridique totalement contrôlée par les insiders;

- un achat de biens et services surfacturé par une entité contrôlée par les insiders (peut être une des holdings de tête dans une construction pyramidale);

- la rémunération de personnes non qualifiées mais proches des insiders;

- la sur-rémunération des personnes compétentes et proches des insiders;

- le sur-investissement dans des projets ne maximisant pas la richesse de l'ensemble des actionnaires.

Dans une perspective de prise de contrôle, la définition du bénéfice privé est élargie de manière à comprendre les effets de synergie, dans la mesure où l'acquéreur de l'entreprise n'en fait pas bénéficier les actionnaires de la cible ${ }^{16}$. Une modification des méthodes de gestion peut être créatrice de valeur.

\subsection{One-share-one-vote}

Grossman et Hart (1988) ou Harris et Raviv (1988), démontrent en retenant des hypothèses somme toute assez raisonnables, la supériorité du système consistant à répartir des droits de vote à raison d'un vote pour chaque action et àn'émettre qu'une seule catégorie d'actions. Par supériorité on entend le choix de l'équipe de direction la plus efficace, c'est-à-dire celle qui crée le plus de valeur pour l'ensemble des actionnaires. Supposons que la valeur de l'entreprise soit constituée :

- d'une part publique (représentée par le flux de dividendes à destination des actionnaires) et

- d'une part privée (dont seuls les dirigeants et/ou les actionnaires exerçant le contrôle peuvent bénéficier).

Dans l'hypothèse où l'entreprise émet plusieurs classes d'actions avec des droits de votes différenciés, un prédateur pourra obtenir le contrôle (la moitié des droits de vote) par une offre publique en acquérant moins de la moitié du droit au flux dividendes. Si l'offre publique doit porter sur la totalité des titres d'une classe donnée (contrainte légale ou statutaire), il prendra le contrôle en acquérant $100 \%$ ou moins du flux de dividendes.

Parmi tous les prédateurs potentiels, celui étant en position de dégager les bénéfices privés (private benefits) les plus forts sera avantagé, même si sa gestion l'amène après la prise de contrôle à dégager le flux public le plus faible. Un rival dont la gestion amènera un fort flux public et un faible flux privé pourra voir son offre dominée par celle du prédateur àfort flux privé et faible flux public.

\footnotetext{
15 "Corporate governance is, to a large extent, a set of mechanisms through which outside investors protect themselves against expropriation by the insiders(...) In general, expropriation is related to the agency problem described by Jensen and Meckling (1976), who focus on the consumption of "perquisites" by managers from the firm's profits. It means that the insiders use the profits of the firm to benefit themselves rather than return the money to the outside investors", La Porta, Lopez-de-Silanes, Shleifer and Vishny (1999).

16 .voir par exemple Grossman et Hart, 1998, p 177.
} 
Ainsi toute déviation du principe «àune action un et un seul droit de vote » occasionnera deux types d'erreurs :

- certains transferts de contrôle seront rendus possibles bien que non souhaitables pour l'ensemble des actionnaires;

- certains transferts de contrôle souhaitables pour l'ensemble des actionnaires seront contrariés.

Grossman et Hart démontrent de manière rigoureuse que ces deux types de risques sont soit réduits dans l'hypothèse où des offres restreintes sont possibles (l'offre publique peut ne porter que sur une fraction du capital ${ }^{17}$ ), soit éliminés dans l'hypothèse où les offres restreintes sont interdites ${ }^{18}$. Harris et Raviv (1988) arrivent à la conclusion qu'un droit de vote et un seul associé avec une règle de prise de contrôle avec la majorité des droits de vote amène à la maximisation de la valeur de l'entreprise.

En fait on peut construire des cas où l'écart au principe « one-share-one-vote » augmente la valeur globale de l'entreprise, en combinant plusieurs des conditions suivantes :

- plusieurs prédateurs en rivalité pensent pouvoir extraire des bénéfices privés conséquents (Hart, 1995, p. 200)

- l'influence des actionnaires n'est pas marginale, ce que l'on peut modéliser en spécifiant un nombre fini d'actionnaires : par exemple, on introduit alors le concept d'actionnaire pivot (Bagnoli et Lipman, 1988);

- l'imposition qui peut affecter différemment la part publique et la part « privée »

- l'offre peut porter sur une partie des titres (restricted offer), une possibilité est alors d'imposer une « super-majority-voting-rule », où la prise de contrôle nécessite le contrôle de plus de 50\% du capital (Grossman, Hart, 1988 ; Harris et Raviv, 1988, 1989). Stulz (1988, p 47) remarque justement que si les règles de super-majorité peuvent être ponctuellement efficaces, leur grand inconvénient, non modélisé, est qu'il est difficile de revenir à la situation antérieure lorsque les actionnaires l'estimeront souhaitable!

- des offres publiques conditionnelles sont obligatoires (l'achat est conditionné à la réussite de l'offre).

En l'absence de bénéfices privés, la répartition des droits de vote sur plusieurs catégories de titres ne pose pas réellement de problèmes, c'est également le cas si les droits de vote sont négociables séparément des droits aux flux financiers. Dans ce dernier cas on distribue à chaque actionnaire un droit aux flux et un droit de vote les deux étant dissociables et pouvant être négociés séparément.

Grossman et Hart (1988) distinguent en fait deux situations dans la conclusion de leur article : la première concerne l'admission en bourse d'une société avec plusieurs catégories d'actions à droits de vote différents; la deuxième concerne les modifications de répartition des votes dans le cas d'une société cotée. C'est la seconde situation qui apparaît la plus susceptible de refléter une tentative d'enracinement des dirigeants et une manouvre de ces derniers en vue d'échapper à tout jugement et sanction de la part du marché du contrôle. Ils suggèrent que la modification des statuts pour une société cotée, notamment en ce qui concerne la répartition des droits de vote, ne puisse être adoptée qu'à une très large majorité.

L'aspect redoutablement efficace du marché du contrôle réside dans le fait que le petit porteur pourra augmenter ses gains et sa satisfaction en joignant ses voix àcelles de la direction de l'entreprise assaillante.

\footnotetext{
${ }^{17}$ Ce qui fait notamment référence à leur proposition 2.

${ }^{18}$ Se reporter àleur proposition 1, page 191.
} 


\subsection{Surveillance et structure de l'actionnariat}

Divers mécanismes autres que la structure de l'actionnariat permettent d'exercer un contrôle sur les dirigeants : le marché du travail (Fama,1980); les banques; les marchés financiers (Easterbrouck, 1984); les marchés des produits (Hart, 1983); et bien sûr le marché du contrôle (Jensen et Ruback, 1983). Toutefois, la répartition des droits de vote, l'éparpillement ou au contraire la présence d'un ou de plusieurs grands actionnaires sont autant d'arguments ayant fait l'objet d'un intense débat. Des argumentations quelquefois contradictoires ont été développées :

- la concentration favorise la surveillance (monitoring), et la dispersion de l'actionnariat accroît la marge de manouvre des dirigeants. Ainsi pour Bhide (1993), la liquidité, associée à la dispersion de l'actionnariat, a un coût;

- la dispersion favorise la surveillance en rendant possible l'OPA. Pour Holmström et Tirole (1993) les informés tirent profit de la présence des bruiteurs dans l'hypothèse d'une information coûteuse : les bruiteurs valorisent le travail de recherche d'informations sur les sociétés cotée;

- la présence d'un grand actionnaire facilite le contrôle. En effet le contrôle par des petits porteurs est limité notamment en raison d'un problème de passager clandestin (Easterbrook et Fischel, 1983). Le grand actionnaire peut lancer une offre publique hostile (Grossman et Hart, 1988);

- La présence d'un "grand" actionnaire facilite le succès d'OPA en cas d'équipe dirigeante inefficace. Elle augmente la valeur des actions et réduit la prime offerte (Shleifer et Vishny, 1986). La concentration de l'actionnariat et la présence d'un actionnaire de référence "non management insider", facilite le contrôle des dirigeants (Demsetz, 1986);

- Le degré d'implication des dirigeants dans le capital des sociétés cotées est un facteur de réduction des conflits entre dirigeants et actionnaires. Il affecte la probabilité de succès d'une offre publique hostile (Mikkelson et Partch, 1989). Pour Morck, Shleifer et Vishny (1986) un fort degré d'implication des dirigeants dans le capital augmente la probabilité de succès de l'offre hostile.

\subsection{Le droit}

Dans un monde sans frictions «à la Modigliani-Miller », les flux sont répartis strictement proportionnellement à la participation aux capitaux propres de l'entreprise et une modification de la structure de l'actionnariat ne change rien àcette répartition.

La prise en compte du pouvoir des investisseurs vis à vis des « insiders » c'est à dire des dirigeants et actionnaires exerçant le contrôle s'appuie sur la distinction entre les droits définis contractuellement et l'inévitable zone de flou, permettant àcelui qui exerce le contrôle effectif de prendre certaines décisions non définies explicitement par le contrat (residual control rights, Hart, 1995).

\subsubsection{Définition des droits de propriété}

Pour Coase (1961) les cas de faillite ou d'échec du marché sont liés à la définition des droits de propriété. Il part du principe qu'une claire définition est nécessaire et suffisante pour retrouver un équilibre avec le marché. Arrow (1979) estime que les droits de propriété sont UNE condition parmi d'autres : un grand nombre d'acheteurs et de vendeurs, la concavité du lieu des opportunités de production, le fait que certains des acheteurs soient informés sur la valeur du bien. On peut également ajouter le coût nécessaire à engager pour faire respecter ses droits contractuels. 
Mais surtout, toutes les situations ne peuvent être contractuellement définies. Ces décisions où le pouvoir s'exerce peuvent concerner : le changement de la direction, la modification de la politique de distribution des dividendes, l'arrêt d'un projet, ou toute décision ayant des effets financiers différents d'une classe d'actionnaire àl'autre (insiders versus outside investor) Le risque d'expropriation peut être limité en supposant des émetteurs et des investisseurs suffisamment sophistiqués pour reconnaître les possibilités d'expropriation des minoritaires et leurs conséquences financières. Les entreprises seront pénalisées et les émetteurs se restreindront d'eux mêmes pour avoir un accès à de meilleures conditions au marché (Jensen et Meckling, 1976). Dans ce contexte la régulation du marché doit être réduite au minimum.

\subsubsection{Régulation du marché}

Pour La Porta, Lopez-de-Silanes, Shleifer, and Vishny (LLSV), 1998, ce sont les contraintes légales, les réglementations et leur application qui sont essentielles àdéfinir les droits de propriété. Ils soutiennent une causalité entre l'efficacité de la protection juridique des investisseurs minoritaires (outside investors) et le développement des marchés financiers. Si leurs intérêts sont protégés, alors les investisseurs deviendront actionnaires. En revanche si ces intérêts sont mal défendus, le gouvernement d'entreprise sera de médiocre qualité et le financement par appel au marché sera moins développé ${ }^{19}$.

LLSV distinguent et opposent l'approche anglo-saxone (common law) donnant une large place à la jurisprudence à notamment l'approche française (civil law) qui accorde plus de place aux textes. Ils soutiennent l'idée que le premier contexte est plus réactif et plus efficace dans la défense des intérêts des actionnaires minoritaires.

La question de savoir si une approche contractuelle est suffisante ou bien si une régulation de marché forte est nécessaire peut difficilement être tranchée d'un point de vue théorique. Mais des implications empiriques sont énonçables et testables. Elles concernent :

- La structure de l'actionnariat qui devrait être plus concentrée dans les pays où la protection des actionnaires minoritaires (outside investors) est moins bien assurée;

- Le lien entre la concentration de l'actionnariat et le système légal de référence (système de type «common law », de type napoléonien, de type allemand avec le code mis en place par Bismarck)...

- Le lien entre la concentration de l'actionnariat, la défense des minoritaires et l'importance du financement externe des entreprises;

- Le lien entre la taille des marchés financiers, relativement à l'importance des économies, et le degré de protection des minoritaires;

- les politiques de distribution de dividende, dans la mesure où un dividende important permet aux actionnaires d'exercer une pression sur les dirigeants et constitue un des outils de contrôle (Jensen et Meckling, 1976) : dans ce cas les politiques de distribution devraient être plus généreuses si les droits des minoritaires sont moins bien assurés.

\section{Evidence empirique : répartition des droits de vote et efficacité économique}

La connaissance de la structure de l'actionnariat est une première étape pas si évidente que cela à franchir. Ensuite il convient de s'interroger sur le lien entre la valeur de la firme et la structure de l'actionnariat. Une autre approche consiste à estimer la valeur et les déterminants

19 voir aussi la conférence donnée par à la Banque Mondiale par La Porta, Lopez-de-Silanes, Shleifer and Vishny (1999). 
des droits de vote. D'autres études enfin, établissent des corrélations entre la répartition des droits de vote ou le degré de protection des actionnaires minoritaires et des indicateurs de l'efficacité économique.

\subsection{Structure de l'actionnariat}

Dans une première section (3.1.1) on rappelle les principaux résultats des études ayant retracé précisément la structure de l'actionnariat des entreprises. La deuxième section (3.1.2) retrace les déterminants de la structure de l'actionnariat.

\subsubsection{Géographie de l'actionnariat}

La dispersion de l'actionnariat est un mythe. Selon La Porta et Lopez de Silanes (1998) aux Etats-Unis, les trois premiers actionnaires détiennent en moyenne $12 \%$ du capital; le chiffre est de $24 \%$ en France; de $50 \%$ en Allemagne, et de $60 \%$ en Italie.

Les études de la structure de l'actionnariat se heurtent à deux difficultés : la première est la définition même du contrôle, la deuxième est la difficulté d'accès aux données (paragraphe 3.1.1.1). Les résultats les plus détaillés concernant la structure de l'actionnariat des entreprises européennes sont présentés par Faccio et Lang (2000), ils sont résumés dans le paragraphe 3.1.1.2. Le paragraphe 3.1.1.3 synthétise les autres études donnant la géographie de l'actionnariat. Le dernier paragraphe conclut.

\subsubsection{Définition du contrôle}

Plusieurs définitions du contrôle sont possibles en fonction de l'objectif poursuivi. Par exemple en France pour définir les conditions de consolidation des comptes : on parle de contrôle de droit, de fait et de contrôle contractuel (voir Bloch et Kremp, 1997). Si l'objectif poursuivi est l'identification de l'actionnaire contrôlant, cela suppose :

1. de connaître la répartition des droits de vote, et dans le cas français de tenir compte des droits de vote double (non recensés par exemple dans la base Dafsaliens qui est une des rares sources publiques concernant les données françaises);

2. de remonter les chaînes de participations sans s'arrêter au premier niveau;

3. de retenir le niveau de participation le plus faible dans une chaîne de droits de vote.

Pour illustrer ces points méthodologiques prenons un exemple italien proposé par Faccio et Lang (2000). En supposant que les liens de la Figure 1 identifient les droits de vote, Compart apparaît comme l'actionnaire en dernier ressort de Montedison, si on retient la définition donnée par la directive européenne ${ }^{20}$. Comme le font remarquer Faccio et Lang il est nécessaire de remonter la chaîne des participations et d'introduire un seuil de prise en compte des participations. Au seuil de $20 \%$ l'actionnaire de référence de Montedison est bien Compart, mais au seuil de $10 \%$ il s'agit de Mediobanca qui détient $3,77 \%+15,26 \%=19,03 \%$ (en retenant le maillon faible de la chaîne). En suivant le même raisonnement, Compart est actionnaire (indirect) d'Edison à hauteur de 33,45\% (et non de $52,65 \%$ si on suit la directive européenne).

L'estimation des participations en capital nécessite en revanche de multiplier les pourcentages de participations intermédiaires (si A détient $15 \%$ de B qui détient lui-même $20 \%$ de C et en supposant une identité entre participation au capital et droits de vote, alors A possède $3 \%$ de $\mathrm{C}$, mais son pouvoir en termes de droits de vote est de $15 \%$ sur C).

\footnotetext{
${ }^{20}$ Voir la directive 88/627/EEC, article 8; Becht (1997) et les travaux du ECGN (European Corporate Governance Network).
} 


\section{Figure 1}

\section{Structure de l'actionnariat de Montedison}

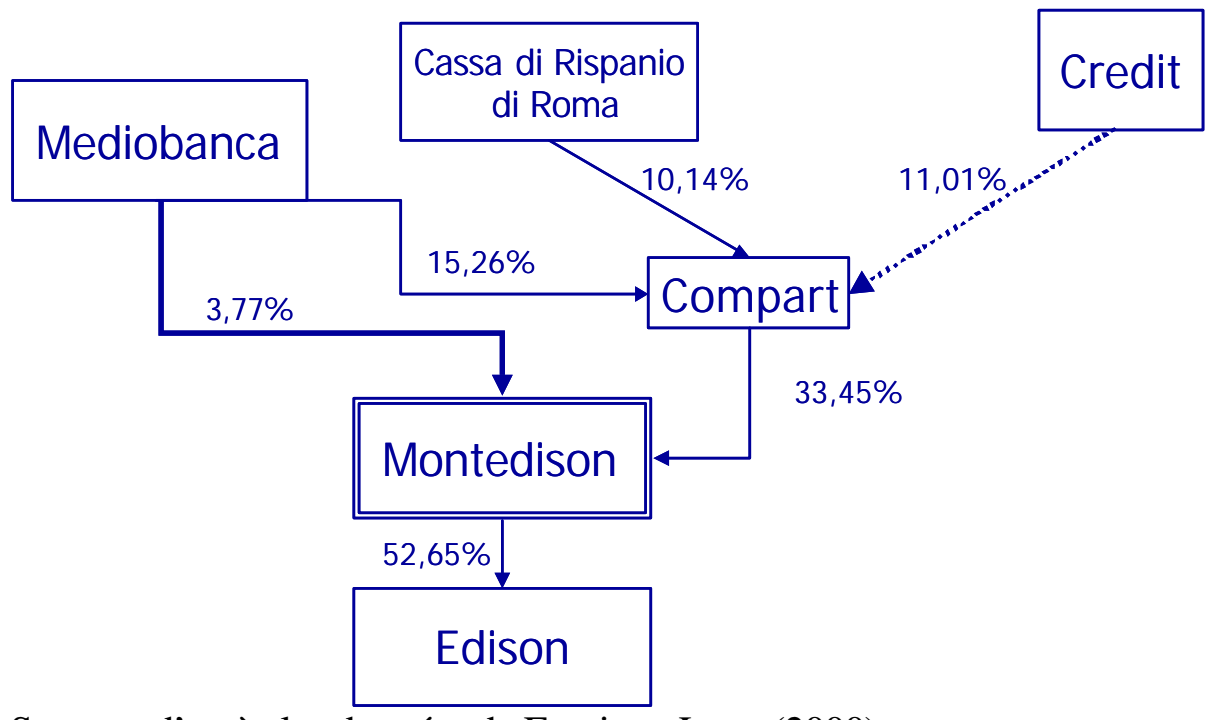

Source : d'après les données de Faccio et Lang (2000)

\subsubsection{Structure de l'actionnariat en Europe}

Faccio et Lang (2000) reconstituent la géographie de l'actionnariat de 3740 entreprises européennes.

Tableau 1 : Actionnaire en dernier ressort des entreprises européennes

\begin{tabular}{|lr|rrr|rrr|}
\hline Pays & Nombre & \multicolumn{3}{|c|}{ Au seuil 10\% : contrôle de type } & \multicolumn{3}{|c|}{ Seuil 20\% : contrôle de type } \\
\cline { 3 - 8 } & entreprises & Dispersé & Familial & Etatique & Dispersé & Familial & Etatique \\
\hline France & 607 & $6,26 \%$ & $70,44 \%$ & $5,17 \%$ & $14,00 \%$ & $64,83 \%$ & $5,11 \%$ \\
Allemagne & 704 & $4,40 \%$ & $71,64 \%$ & $5,23 \%$ & $10,37 \%$ & $64,62 \%$ & $6,30 \%$ \\
Italie & 208 & $5,29 \%$ & $64,87 \%$ & $9,98 \%$ & $12,98 \%$ & $59,61 \%$ & $10,34 \%$ \\
Grande- & 1589 & $26,18 \%$ & $33,75 \%$ & $0,19 \%$ & $68,09 \%$ & $19,88 \%$ & $0,09 \%$ \\
Bretagne & & & & & &
\end{tabular}

Note : ces résultats sont tirés de Faccio et Lang (2000), table 2. En France sur 607 entreprises étudiées en décembre 1996, 6,26\% ont un contrôle de type dispersé au seuil de 10\%;70,44\% des entreprises sont contrôlées par une «famille », ce qui référence à un actionnaire lui même non coté; et 5,17\% sont contrôlées par l'état toujours au seuil de $10 \%$. La somme de ces trois pourcentage est inférieure à 100\% car d'autres catégories, non reportées ici, sont prises en compte par les auteurs. Les pourcentages sont significativement différents entre la GrandeBretagne et les autres pays dans chaque colonne du tableau.

Le Tableau 1 retrace le type de contrôle des entreprises européennes en retenant un seuil de 10\% (voir la section 3.1.1.1), il permet de constater :

1. l'importance du contrôle de type familial;

2. la grande différence entre la Grande Bretagne et les autres pays européens quant à la proportion d'entreprise au contrôle dispersé;

3. Le seuil de $20 \%$ au lieu de $10 \%$, augmente la proportion d'entreprises dont le contrôle est dispersé et réduit la proportion des entreprises à contrôle familial. Les différences entre les deux seuils sont surtout sensibles dans le cas de la Grande Bretagne.

Une analyse plus fine des résultats permet aux auteurs de montrer qu'en France 33,80\% de la capitalisation des entreprises de l'échantillon, qui comprend 607 entreprises cotées, est détenu 
par les 15 premières familles, contre 5,46\% en Grande-Bretagne ou 21,92\% en Italie. Les cinq premières familles contrôlent $22,04 \%$ de la somme des capitalisations des entreprises françaises cotées, contre 3,79\% en Grande-Bretagne ou 16,83\% en Italie. Les liens entre les familles et le monde politique sont soulignés par les auteurs notamment dans le cas de l'Italie et de la France (voir Faccio et Lang, 2000, en particulier page 21).

Par ailleurs, la proportion d'entreprises à contrôle familial est plus importante pour les entreprises de petite taille, ce qui n'est pas très étonnant : ainsi dans le cas de la France $88,93 \%$ des cinquante plus petites entreprises sont contrôlées par une famille au seuil de $10 \%$ contre $50 \%$ des vingt entreprises les plus grandes.

Les mécanismes mis en ouvre pour s'assurer du contrôle des entreprises sont assez différents d'un pays à l'autre : l'Italie se singularise par le recours aux classes d'actions à droits de vote multiple (41,35\% des cas); les constructions pyramidales sont surtout utilisées en Italie (26,90\% des cas), en Allemagne puis en France (17,75\% des cas). Faccio et Lang ne disposent pas de la base de données idéale puisque tous les mécanismes de dissociation entre droits de vote et apports en capital ne sont pas identifiés dans leurs données. En particulier les mécanismes de droits de vote double aux actions ordinaires sous condition de détention ne sont pas pris en compte dans le cas de la France. Les cas d'un actionnaire dominant sans deuxième actionnaire à plus de $10 \%$ sont les plus fréquents en France $(64,85 \%)$. Le rôle de surveillance (monitoring) du second actionnaire n'est donc pas rempli et le risque d'expropriation ou de spoliation des minoritaire est alors potentiellement plus important. Les auteurs montrent enfin la fréquence extrêmement importante des situations où un membre de la famille dominante est membre de la direction de l'entreprise. Les résultats minorent probablement la réalité puisque les auteurs ont identifié les situations à partir d'un relevé des noms identiques, sans retracer les liens familiaux effectifs.

\subsubsection{3. $\quad$ Autres études}

Des études récentes comparent les structures de l'actionnariat de différents pays et mettent en évidence de grandes différences (Etudes générales). D'autres s'intéressent à la structure du capital des entreprises récemment introduites en bourse, à la structure de l'actionnariat des entreprises à droit de vote plural, au vote des investisseurs institutionnels, ou àl'efficacité des batailles de mandats. Ces différents thèmes sont successivement développés ci après.

\section{- Etudes générales}

Pour la Security and Exchange Commission, dans une étude de 1984 sur 6000 entreprises cotées aux Etats-Unis, dans 15\% des cas un dirigeant détient au moins 10\% du capital. La Porta, Lopez-de-Silanes, Shleifer et Vishny (1999) retracent la structure de l'actionnariat et identifient l'actionnaire en dernier ressort de trente entreprises cotées dans 27 pays : les vingt plus grandes et dix qualifiées de moyennes. Leur étude est menée sur des données entre 1995 et 1997 et retrace tous les actionnaires détenant $10 \%$ ou plus des droits de vote.

Si les vingt plus grandes entreprises cotées au Japon, aux Etats-Unis ou en Grande-Bretagne sont effectivement caractérisées très majoritairement par un actionnariat dispersé, tel n'est pas la situation rencontrée dans tous les pays (se reporter au Tableau 2) : le contrôle de l'Etat et le contrôle familial jouent un rôle déterminant.

Pour les dix entreprises dans chaque pays que les auteurs qualifient de moyennes en taille (ce sont les dix plus petites avec une capitalisation boursière d'au moins 500 millions de dollars en 1995), les résultats sont sensiblement différents et rejettent plus nettement l'hypothèse d'un actionnariat dispersé. 
Tableau 2 : Structure de l'actionnariat dans les entreprises cotées

\begin{tabular}{|l|c|c|c|c|c|c|}
\hline Pays & $\begin{array}{c}\text { Actionnariat } \\
\text { dispersé }\end{array}$ & Familial & Etat & $\begin{array}{c}\text { Dispersé } \\
\text { financières }\end{array}$ & $\begin{array}{c}\text { Dispersé } \\
\text { entreprises }\end{array}$ & Autres \\
\hline Japon (grandes) & 0.90 & 0.05 & 0.05 & 0.00 & 0.00 & 0.00 \\
\hline Etats-Unis (grandes) & 0.80 & 0.20 & 0.00 & 0.00 & 0.00 & 0.00 \\
\hline Etats-Unis (moyennes) & 0.90 & 0.10 & 0.00 & 0.00 & 0.00 & 0.00 \\
\hline Grande Bretagne (grandes) & 1.00 & 0.00 & 0.00 & 0.00 & 0.00 & 0.00 \\
\hline Grande-Bretagne (moyennes) & 0.60 & 0.40 & 0.00 & 0.00 & 0.00 & 0.00 \\
\hline France (grandes) & 0.60 & 0.20 & 0.15 & 0.05 & 0.00 & 0.00 \\
\hline France (moyennes) & 0.00 & 0.50 & 0.20 & 0.20 & 0.00 & 0.10 \\
\hline Italie (toutes) & 0.20 & 0.15 & 0.40 & 0.05 & 0.10 & 0.10 \\
\hline Allemagne (toutes) & 0.50 & 0.10 & 0.25 & 0.15 & 0.00 & 0.00 \\
\hline
\end{tabular}

Note : extrait et synthèse des données des tableaux 2 et 3 de La Porta, Lopez-de-Silanes, Shleifer et Vishny (1999), le contrôle est défini avec un seuil de $20 \%$

La dissociation entre la participation au capital et les droits de vote, les structures pyramidales et les participations croisées sont également des phénomènes plus fréquents en Europe continentale que par exemple au Japon, en Grande Bretagne ou aux Etats-Unis dans les grandes entreprises cotées suivant La Porta, Lopez-de-Silanes, Shleifer et Vishny (1999). Claessens, Djankov et Lang (2000) ont compilé la structure de l'actionnariat de 2980 entreprises de neuf pays asiatiques (dont le Japon, Hong Kong, la Malaisie et Taiwan). Ils mettent en évidence le rôle joué par les structures pyramidales et les participations croisées et surtout le fait que les deux tiers des entreprises sont contrôlées par un seul actionnaire. Les dirigeants sont souvent proches des familles détenant le contrôle (plusieurs auteurs parlent de « crony capitalism »). Les firmes anciennement cotées sont le plus souvent contrôlées par une famille. En moyenne le principal actionnaire détient $6,90 \%$ du capital et $10,33 \%$ des droits de vote au Japon, ces chiffres sont de $24,3 \%$ et $28,08 \%$ à Hong Kong, de 23,89\% et 28,32\% en Malaisie et de $15,98 \%$ et $18,96 \%$ à Taiwan.

\section{- Structure du capital des entreprises récemment introduites en bourse}

De Angelo, De Angelo (1985) observent que les dirigeants détiennent en médiane 50,9\% du capital des entreprises introduites en bourse aux Etats-Unis.

\section{- Structure de l'actionnariat des entreprises à droit de vote plural}

De Angelo et De Angelo (1985) étudient la structure de l'actionnariat de 45 entreprises américaines ayant émis des titres à droits de vote différenciés. Dans tous les cas, les dirigeants détiennent une participation importante dans la société (en moyenne 56,9\% des droits de vote et $24 \%$ des droits aux flux financiers); dans 27 cas sur 45 la direction détient le contrôle absolu, dont seize cas pour lesquels le contrôle est réalisé par une personne; dans quinze entreprises plusieurs membres de la famille qui détient le contrôle figurent dans les cinq salaires les plus élevés payés par la société. Quatre prises de contrôle sont détaillées dans lesquelles les détenteurs de blocs de contrôles avec forts droits de vote ont touché une prime conséquente, valorisant le droit de vote entre $80 \%$ et $200 \%$ de la valeur de l'action sans droit de vote.

\section{- Le vote des investisseurs institutionnels}

Brickley, Lease et Smith Jr (1988) examinent le lien éventuel entre l'actionnariat institutionnel et le vote de diverses dispositions limitant ou contrariant les offres publiques ${ }^{21}$.

\footnotetext{
${ }^{21}$ Les dispositions sont les suivantes : règle de super-majorité sur les offres publiques n'ayant pas l'agrément des dirigeants en place. Création de nouvelles catégories d'actions privilégiées. Mise en place d'un renouvellement fractionné du conseil d'administration (classify board). Règles de super-majorité sur les fusions. Clauses limitant le remplacement de dirigeants, ne pouvant être remplacés que pour « just cause ». Localisation juridique dans un
} 
Les résultats permettent aux auteurs de définir deux catégories d'investisseurs institutionnels : les banques, les compagnies d'assurances et les trusts d'une part et d'autre part les mutuals funds, fondations et fonds de pension. Ceux appartenant à la deuxième catégorie s'opposent plus fréquemment aux dirigeants en place.

\section{- Efficacité des batailles de mandats}

Les batailles de mandats (proxy fights) sont un des moyens pour les actionnaires de faire valoir leurs droits. Entre 1981 et 1984 aux Etats-Unis, Pound (1988) dénombre une centaine de batailles de mandats dont 60 concernaient le contrôle de l'entreprise contre 250 offres publiques (tender offer) sur des entreprises cotées en bourse. L'étude empirique confirme une relative inefficacité de ce type de procédure :

$\circ$ qui donne un avantage à la direction en place;

- dans lesquelles le vote des investisseurs institutionnels et intermédiaires détenant les titres (fiduciaries) est plutôt acquis aux directions en place indépendamment des intérêts des actionnaires;

- ce type de procédure ne permet pas de signaler clairement les intentions du groupe contestant le contrôle : le faible coût apparent, l'absence d'une prime de contrôle offerte aux minoritaires, ne permettent pas de lever la méfiance et de discriminer entre les offres (problème d'anti-sélection).

\subsubsection{Conclusion}

La vision de Berle et Means avec d'un coté les dirigeants et de l'autre les actionnaires atomisés ne recoupe pas les observations faites. Dans beaucoup de pays le contrôle familial des entreprises est une réalité. LLS (1999) ou Claessens et al (2000) montrent que le plus souvent les entreprises contrôlées par une famille sont également gérées par un membre de la famille ce qui réduit encore la place envisagée par Berle et Means pour les dirigeants professionnels. Dans de nombreux pays le problème d'agence n'est pas celui proposé par Berle et Means et qui oppose les dirigeants (professionnels) et les actionnaires, mais plutôt est la conséquence d'une opposition entre les actionnaires minoritaires (outside investors) et ceux aux affaires, ces derniers ayant un contrôle presque total sur les dirigeants.

\subsubsection{Déterminants de la structure de l'actionnariat}

Pour quelles raisons en Europe continentale ou en Asie le contrôle familial est-il si important? Quels sont les déterminants des structures de l'actionnariat d'un pays àl'autre?

\subsubsection{Concentration de l'actionnariat et valeur de l'entreprise} ou la préférence du marché pour la dilution

De nombreuses études tendent à montrer un lien négatif entre la valeur de marché des capitaux propres et la concentration de l'actionnariat. Cette interprétation est notamment justifiée par les observations suivantes :

- le décès d'un actionnaire majoritaire est accompagné d'une augmentation de la valeur de l'entreprise, d'une réduction de la concentration de l'actionnariat et d'une augmentation de la probabilité d'OPA (Slovin et Sushka,1993) : lorsque l'actionnaire décédé détient $10 \%$ ou plus, l'annonce de son décès a un impact positif sur la valeur des actions. Ce résultat est interprété comme une validation de l'hypothèse d'enracinement des dirigeants (managerial entrenchment hypothesis). En effet après 
un décès, généralement on assiste à une dispersion du capital détenu. Ce qui augmente la probabilité d'offres hostiles ou amicales dans la majorité des cas (Stulz 1988);

- si les dirigeants (insiders) détiennent un faible pourcentage du capital, l'accroissement de leur participation accroît la valeur de la firme, mais au fur et à mesure que le niveau de participation augmente, tout accroissement réduit la valeur de la firme. Morck, Shleifer et Vishny (1988) montrent à partir d'une étude portant sur 456 entreprises en 1980, un lien entre le pourcentage de capital détenu par les dirigeants et le ratio Q de Tobin (ratio entre la valeur de marché de la firme au numérateur et le coût de remplacement des actifs productifs au dénominateur). Les auteurs interprètent le résultat comme une évidence de l'amélioration de l'efficacité de la gestion des entreprises (du point de vue des actionnaires) tant que le pourcentage détenu par les dirigeants ne dépasse pas un certain seuil. Les résultats ne sont toutefois pas sans ambiguï tés.

- Tnani (1997), étudie la réaction du marché à 979 annonces de franchissements de seuils à la bourse de Paris, réparties entre janvier 1988 et décembre 1990. Elle obtient différents résultats originaux : 1) le délai d'annonce est supérieur à 15 jours dans $42,74 \%$ des cas. Le délai moyen d'annonce au marché du franchissement de seuil est de 23 jours et il s'allonge après la loi de 1989 qui réglemente pourtant plus sévèrement les obligations de publication en la matière. 2) Elle observe une asymétrie des réactions du marché aux franchissements de seuils à la hausse et à la baisse. Plus précisément une augmentation des cours est constatée lors de franchissements de seuils à la baisse. L'auteur conclut à la préférence du marché pour la dilution.

\subsubsection{Protection des actionnaires et géographie de l'actionnariat}

L'indicateur de protection des droits des actionnaires minoritaires de La Porta et Lopez de Silanes (1998), présenté ci-dessus, est repris dans diverses études notamment concernant le lien entre les droits des actionnaires minoritaires et la structure de l'actionnariat.

Tableau 3 : Structure de l'actionnariat et droits des actionnaires minoritaires

\begin{tabular}{|l|c|c|c|c|c|c|}
\hline & $\begin{array}{c}\text { Actionnari } \\
\text { at dispersé }\end{array}$ & Familial & Etat & $\begin{array}{c}\text { Dispersé } \\
\text { financières }\end{array}$ & $\begin{array}{c}\text { Dispersé } \\
\text { entreprises }\end{array}$ & Autres \\
\hline & \multicolumn{7}{|c|}{ Entreprises de grande taille } \\
\hline Forte protection & 0.4792 & 0.2458 & 0.1375 & 0.0250 & 0.0833 & 0.0292 \\
\hline Faible protection & 0.1600 & 0.3833 & 0.2367 & 0.1100 & 0.0200 & 0.0900 \\
\hline Test de Student & -1.92 & 0.88 & 1.05 & 1.50 & -1.50 & 0.06 \\
\hline & \multicolumn{7}{|c|}{ Entreprises moyennes } \\
\hline Forte protection & 0.3750 & 0.3850 & 0.0867 & 0.0417 & 0.0358 & 0.0775 \\
\hline Faible protection & 0.1267 & 0.5047 & 0.2020 & 0.0400 & 0.0400 & 0.0867 \\
\hline Test Student & -2.86 & 1.24 & 1.64 & -0.45 & 0.18 & 0.16 \\
\hline
\end{tabular}

Note : extrait des données des tableaux 2 et 3 de La Porta, Lopez-de-Silanes, Shleifer et Vishny (1999). En ligne, les 27 pays sont répartis en deux groupes suivant la valeur de l'indicateur de protection des droits des actionnaires minoritaires (anti-director rigths). Les auteurs distinguent les entreprises suivant leur capitalisation boursière (grande ou moyenne) en ligne et suivant le type de contrôle en colonnes.

La Porta, Lopez-de-Silanes, Shleifer et Vishny (1999) montrent que la dispersion du capital est d'abord observée dans les pays où les droits des actionnaires minoritaires sont les mieux défendus. Les auteurs répartissent les 27 pays étudiés en deux groupes d'effectif identique suivant le degré de protection des actionnaires minoritaires (en fait la valeur de la variable « anti director rights »). Le Tableau 3 montre ainsi le lien très net entre le pourcentage d'entreprise dont le capital est dispersé et la protection des minoritaires : en moyenne 47,92\% 
des entreprises de grande taille appartiennent à cette catégorie dans les pays à forte protection (dont les Etats-Unis et la Grande Bretagne), contre 16\% dans les pays où cette protection est moins bien assurée (dont la France, l'Allemagne ou l'Italie). La différence est encore plus significative dans le cas des entreprises cotées de taille moyenne (les dix plus petites de chaque pays ayant une capitalisation supérieure à 500 millions de dollars en 1995).

La Porta et Lopez de Silanes (1998) régressent le pourcentage du capital action détenu par les trois principaux actionnaires des dix plus grandes entreprises cotées dans 47 pays, contre différentes variables caractérisant le pays dans lequel l'entreprise est cotée. L'importance de l'économie mesurée par le produit national brut joue un rôle important pour expliquer les différences de concentration de l'actionnariat des grandes entreprises, mais la variable au poids le plus fort est celle synthétisant la protection des minoritaires : une augmentation de un point de cette variable est associée à une baisse de 3,85\% de la participation des trois principaux actionnaires. Les auteurs interprètent ces résultats de la manière suivante : si l'intérêt des minoritaires est peu ou moins bien protégé, il devient particulièrement important d'exercer un contrôle effectif de l'entreprise et ainsi la forte concentration de l'actionnariat serait une conséquence de la faible protection légale assurée aux actionnaires minoritaires. Ces résultats montrent l'importance de la définition d'un environnement légal exerçant certaines contraintes sur la rédaction des contrats et la définition même des droits de propriété.

\subsection{Valeur des droits de vote}

Quelle est la valeur des droits de vote d'un actionnaire minoritaire? Elle est liée à la probabilité que ces votes jouent un rôle pivot dans une coalition, elle est également plus importante en cas de possibilité d'extraction de bénéfices privés par un groupe d'actionnaires exerçant le contrôle. Comment mesurer la valeur des droits de vote?

- par comparaison des valeurs de deux classes de titres avec droits de vote différents;

- ou en mesurant la réaction du marché lors de modification de règles de répartition des droits de vote.

Quels sont les liens entre la valeur du droit de vote et la possibilité de capture de bénéfices privés? Ces trois thèmes sont successivement étudiés.

\subsubsection{Différence de valeur entre deux catégories d'actions à droits de vote différencié}

Comment mesurer la valeur des droits de vote? Par comparaison de la valeur en bourse de deux types de titres émis par une société et que rien ne distingue sauf des droits de vote différents, on calcule une prime des titres avec droits de vote ou une décote des titres sans. Pour Lease, McConnell et Mikkelson (1983) la décote est en moyenne de 3,8\% pour les entreprises cotées aux Etats-Unis. Pour Levy (1982) la prime est de 45,5\% en Israël, pour Rydqvist (1992) de 6,5\% en Suède, pour Zingales (1994) de $81 \%$ en Italie.

Les résultats pour la France doivent être interprétés avec prudence en raison du faible nombre d'observations et de la grande différence de liquidité entre les titres avec ou sans droit de vote : Husson, Jacquillat et Schintowski (1987), observent une décote moyenne de $20 \%$ (entre $6,4 \%$ et $33,4 \%$ pour 8 certificats d'investissement en 1985-1986).

La décote des actions sans droits de vote est estimée en moyenne égale à $82 \%$ pour les actions cotées à la bourse de Milan entre 1987 et 1990 (Zingales, 1994). Ces actions sans droits de vote (azioni di risparmio) se sont multipliées récemment à la bourse de Milan : autorisées à partir de 1974, il y en avait 6 au début des années 80, 44 nouvelles entre 1985 et 1986 et 64 au total dans l'étude de Zingales (1994). 


\subsubsection{Impact du changement des règles de vote}

Jarrell et Poulsen(1988) s'intéressent aux variations de valeur des titres lorsque les dirigeants d'entreprises modifient certaines règles de répartition des droits de vote. Les «dual class recapitalization » sont l'occasion pour les dirigeants de renforcer la part de droit de vote qu'ils détiennent sans renforcer dans la même proportion et quelquefois même en réduisant leur participation aux capitaux propres. Dans 87 cas où la structure de l'actionnariat a pu être précisément retracée, les insiders détiennent en moyenne 43,7\% des actions (et donc des droits de vote) avant l'opération. Dans 89 cas sur 94 la réaction des actionnaires est négative. La réaction est négative et se traduit par une baisse du cours égale à un peu moins de $1 \%$ en moyenne, surtout pour les opérations réalisées après que le NYSE, en juin 1984, ait imposé un moratoire sur l'exclusion de la cote des entreprises émettant plusieurs classes d'actions de droits de vote différents. La réaction est également surtout négative dans le cas où les insiders détiennent moins de 50\% du capital avant l'opération. Pour les auteurs, ces résultats valident une hypothèse d'enracinement des dirigeants qui utiliseraient ces opérations dans le but d'échapper àdes offres hostiles.

\subsubsection{L'importance des bénéfices privés}

La constatation de très fortes progressions de cours, en quelques séances, pour des sociétés cotées mais contrôlées par l'Etat, et à l'annonce d'une privatisation totale est pour Zingales (1994) un élément compatible avec l'existence de forts bénéfices privés : Credito Italiano voit ainsi le cours de son action progresser de 38\% en 5 séances en septembre 1992. L'interprétation généralement proposée de la décote des actions sans droits de vote par rapport à celles qui en sont pourvues, lie cette dernière à l'importance des bénéfices privés qui seraient donc plus importants en Europe latine qu'aux Etats-Unis. Barclay et Holderness (1989) aux Etats-Unis attribuent la prime payée (environ 4\%) pour l'acquisition d'un bloc d'action à l'existence de bénéfices privés.

La valeur cotée des holdings semble contredire le principe d'additivité des valeurs suivant lequel la valeur de l'ensemble doit être égale à la somme de la valeur des composantes. De nombreuses explications ont été proposées de l'observation d'une décote des holdings. Barclay, Holderness et Pontiff (1993) s'intéressent aux fonds d'investissement àcapital fixe (closed-end funds) pour lesquels une décote est régulièrement documentée dans la littérature financière. Les auteurs répartissent les fonds fermés en deux groupes suivant la structure de l'actionnariat du fonds fermé. Ils documentent une décote de 14\% dans le groupe de fonds ayant des actionnaires détenant une part significative du capital et $4 \%$ seulement de décote si l'actionnariat est dispersé. Ils étudient plusieurs cas de bataille pour l'ouverture de fonds ${ }^{22}$. Une enquête quasiment policière amène les auteurs àidentifier de nombreuses sources d'avantages liés au contrôle de ces fonds fermés.

\subsection{Structure de l'actionnariat et efficacité économique}

Quel est le meilleur système parmi la grande diversité des mécanismes de gouvernement d'entreprise que l'on observe à travers le monde s'interrogent Shleifer et Vishny (1997)? Doit-on préférer le système anglo-saxon que l'on observe en Grande-Bretagne ou aux EtatsUnis, qui protège légalement le plus fortement les droits des actionnaires minoritaires et dans lequel les «grands actionnaires» jouent un rôle moins proéminent, mis à part leurs interventions sporadiques dans les offres publiques. Ou doit-on préférer les mécanismes

\footnotetext{
${ }^{22}$ Ainsi la bataille qui a duré plusieurs années menée par Vanguard contre Liberty All-Star. En 1987, le groupe Vanguard calcule que l'ouverture du fonds permettrait de récupérer 90 millions de dollars et essuie un refus d'ouverture de la part de la direction; Vanguard propose d'assurer la gestion pour un coût annuel de 600.000 dollars (soit un gain de 1,8 million) et essuie évidemment un nouveau refus.
} 
observés en Europe continentale ou au Japon où le rôle des banques et des groupes de contrôle est plus fort?

Le point réellement important, pour la collectivité, est l'efficacité des mécanismes de répartition des droits de vote. Comment en juger? Le numéro spécial d'octobre 2000 du Journal of Financial Economics contient plusieurs contributions décisives sur ces thèmes. Diverses études réalisent des comparaisons internationales et démontrent un lien entre la protection des minoritaires et

- le développement des marchés financiers et plus particulièrement des marchés d'actions et donc l'importance du financement externe des entreprises cotées (section 3.3.1.);

- la diffusion d'informations par les entreprises (section 3.3.2);

- l'efficacité de l'allocation des capitaux et la capacité à les diriger vers les secteurs d'activité en croissance au détriment de ceux en déclin (section 3.3.3).

\subsubsection{Développement des marchés et contribution au financement des entreprises}

La taille et le degré de développement des marchés est très inégal d'un pays àl'autre, même si on tient compte des différences d'échelle des économies. Dans un monde sans frictions, «àla Modigliani et Miller», la taille des marchés ne devrait-elle pas être strictement proportionnelle aux flux générés par l'ensemble des entreprises en direction de leurs propriétaires, s'interrogent La Porta, Lopez-de-Silanes (1998)? Et ainsi ne devrait-on pas observer une taille des marchés proportionnelle aux produits intérieurs bruts (PIB)? En fait, on observe que :

- le ratio Capitalisation/PIB est très différent d'un pays àl'autre. Ainsi, le rapport entre le PIB de l'année 1998 et la capitalisation des actions nationales à la fin de la même année est de 50,9\% en Allemagne, 67,8\% en France et de 148,6\% aux Etats-Unis ou $171 \%$ en Grande Bretagne (à partir des données de la FIBV) ;

- la structure du portefeuille des ménages est également différente : en 1998, le portefeuille des ménages français accorde une place de $32 \%$ aux placements monétaires et $18 \%$ aux placements en actions. La même année, la part placée en actions est de $83,5 \%$ en Grande Bretagne, $75 \%$ en Suède ou $69,8 \%$ en Suisse.

Une conséquence directe du degré de développement des marchés est leur contribution au financement des entreprises. L'importance du recours au financement externe d'un pays à l'autre dépend notamment des mesures de défense des actionnaires minoritaires (antidirector-rights), ainsi l'étude de La Porta et Lopez de Silanes (1998), le met en évidence.

\subsubsection{Diffusion d'informations et protection des minoritaires}

Morck, Yeung et Yu (2000) distinguent de manière assez classique deux types d'informations susceptibles d'affecter les cours des actions :

- les informations qui touchent toutes les actions, chacune répondant avec une sensibilité qui lui est propre (changement de politique monétaire, publications d'informations macro économiques, projets de modification de la fiscalité...)

- et les informations spécifiques à une entreprise, qui n'affecteront qu'une ou un petit ensemble d'entreprises (publications des prévisions de bénéfices, annonces concernant les produits...).

Dans l'hypothèse où le premier type d'information domine, les cours des différentes actions auront tendance à bouger dans le même sens. Dans l'hypothèse en revanche où la part 
d'information spécifique augmente, relativement àl'information «commune», les mouvements de cours sur un intervalle donné sont moins bien synchronisés. Morck, Yeung et Yu (2000) s'intéressent ainsi à la corrélation entre les taux de rentabilités des différentes actions cotées sur une place boursière donnée, ils observent :

1. une baisse aux Etats-Unis, de la corrélation des variations relatives de cours constatée entre toutes les actions cotées, depuis le début du XXè siècle, ce qui accrédite l'idée d'une plus grande quantité d'information privée ou spécifique produite, relativement à l'information commune ou macro-économique ;

2. de très grandes disparités, d'un pays à l'autre en 1995 : alors que les corrélations intertitres sont faibles entre les entreprises cotées aux Etats-Unis, cette corrélation est beaucoup plus forte sur les marchés des pays moins développés ou très récents.

Mais comment expliquer les différences constatées d'un pays àl'autre entre les valeurs de ce coefficient de corrélation? Quels en sont les déterminants? Morck, Yeung et Yu (2000) réalisent une régression entre les valeurs de ces coefficients de corrélations et différentes caractéristiques des marchés. La variable expliquée est le logarithme de la variance des résidus d'une régression entre les rentabilités des actions et l'indice de référence du pays où les actions sont cotées. Les taux de rentabilité sont bi-mensuels en 1995 et 22 pays sont pris en compte. Parmi les variables indépendantes on privilégiera ici l'indice de protection des actionnaires minoritaires (anti-director rights index). Le $\mathrm{R}^{2}$ de la régression passe de 41 à $63 \%$ et la statistique $\mathrm{F}$ de 1,41 à 3,37 àl'introduction de cette variable qui est hautement significative $^{23}$.

Le message peut être exprimé de la manière suivante : puisque l'activité de production d'informations est coûteuse, la démarche de production d'informations spécifiques est plus intense lorsque les conditions économico-juridiques rendent possible la valorisation de la démarche. Si le capital d'une société est verrouillé, pourquoi dépenser de l'argent en étude précise et en mesure du degré de sous-évaluation de l'entreprise? L'indicateur de Morck, Yeung et Yu (2000) est dans ce sens un indicateur d'efficience ou de degré de révélation des informations dans les cours cotés.

\subsubsection{L'efficacité $\mathrm{d}$ e l'allocation des capitaux}

Wurgler (2000) estime l'efficacité de l'allocation des ressources c'est-à-dire la capacité à attirer les capitaux vers les activités en croissance et à éviter les surinvestissements dans les secteurs en déclin. L'approche retenue tient compte des données effectivement disponibles. L'auteur propose de retenir la sensibilité de l'accroissement de l'investissement à l'accroissement de la valeur ajoutée, plus précisément il mesure le coefficient de la régression suivante :

$$
\operatorname{Ln}\left(\frac{I_{i c t}}{I_{i c t-1}}\right)=\alpha_{c}+\eta \times \operatorname{Ln}\left(\frac{V_{i c t}}{V_{i c t-1}}\right)+\varepsilon_{i c t}
$$

avec I, l'investissement brut (les amortissements ne sont pas disponibles pour beaucoup de pays) estimé pour un secteur (i), un pays (c) et une année (t) donnée. V est la valeur ajoutée d'un secteur (i), d'un pays (c) et une année donnée (t). La pente estime la propension d'un pays donné à accroître les investissements dans les secteurs d'activité en croissance et à les réduire dans les secteurs d'activité en déclin. Wurgler montre à partir de données de 65 pays de 1963 à 1995 que l'efficacité de l'allocation des capitaux ainsi mesurée est :

- négativement corrélée à la participation de l'Etat en tant qu'actionnaire des entreprises;

\footnotetext{
${ }^{23}$ Avec un coefficient estimé à 0.168 et un écart-type de 0.01 . Six autres variables sont introduites dont un indice de concentration des secteurs d'activité (Herfindahl) qui a un coefficient estimé à 0.918 (0.62)
} 
- positivement lié à la quantité d'information spécifique produite (mesurée par le coefficient de corrélation entre les rentabilités des différentes actions cotées suivant la proposition de Morck, Yeung et Yu, 2000);

- et positivement corrélée au degré de protection des intérêts des actionnaires minoritaires. Wurgler (2000) présente certains résultats montrant notamment le rôle de cette variable pour éviter le surinvestissement dans les industries déclinantes, ce qui corrobore l'hypothèse de gaspillage du free-cash-flow formulée par Jensen (1986).

\section{Résumé, conclusions et perspectives}

La vision de Berle et Means avec d'un coté les dirigeants et de l'autre un actionnariat atomisé ne recoupe pas les observations faites. Dans beaucoup de pays le contrôle familial des entreprises est une réalité; le problème d'agence n'y est pas celui proposé par Berle et Means qui oppose les dirigeants (professionnels) et les actionnaires, mais plutôt est la conséquence d'une opposition entre les actionnaires minoritaires (outside investors) et ceux aux affaires (insiders), ces derniers ayant un contrôle presque total sur les dirigeants.

Un des déterminants le plus significatif de la structure de l'actionnariat est la qualité de la protection juridique accordée aux actionnaires minoritaires. Les marchés les plus développés relativement àl'importance de l'économie sont également ceux où les droits des actionnaires minoritaires sont les mieux protégés par la loi ou la réglementation ${ }^{24}$.

Le développement des marchés financiers et de l'intermédiation financière est un moteur de croissance : il stimule l'épargne, favorise la surveillance (monitoring) des entreprises et est ainsi un facteur d'amélioration de leur gestion, il facilite l'allocation des ressources dans l'économie (Wurgler, 2000). Le lien entre le degré de développement des marchés, leur liquidité et la croissance économique a fait l'objet de plusieurs études aux résultats concordants (King et Levine, 1993; Levine et Zervos, 1998; Demirguc-Kunt et Maksimovic, 1998, 1999).

Par ailleurs le développement des marchés s'accompagne d'une production d'informations spécifiques aux entreprises. Morck, Yeung et Yu (2000) montrent le rôle joué par la protection des actionnaires minoritaires dans la production des informations et probablement le degré d'efficience des marchés.

Le rôle de la loi et de la régulation de marché est important dans l'amélioration de la protection des actionnaires minoritaires :

- On peut ainsi suggérer l'introduction de clauses de quorum ou de veto pour toute modification statutaire, notamment celles impliquant une modification des structures de vote, avec la nécessité d'obtenir de nouveau le vote des actionnaires pour tous les dispositifs actuellement opérationnels et contraires au principe « àune action un droit de vote et un seul ».

- L'obligation de déclaration, avec publication, de toute transaction réalisée par les dirigeants d'une entreprise ou par les actionnaires détenant plus de $\mathrm{x} \%$ (10\% aux Etats-Unis), améliorerait la transparence.

Les voies de recherche futures sont nombreuses. On a mentionné la nécessité du développement de bases de données répertoriant et suivant dynamiquement les structures de

\footnotetext{
24 "An important implication of this approach is that leaving financial markets alone is not a good way to encourage them. Financial markets need some protection of outside investors, whether by courts, government agencies, or market participants themselves. Improving such protection is a difficult task. (...) Marginal reform may not successfully achieve the reformer's goals. In part, the existing corporate governance arrangements benefit both the politicians and the entrenched economic interests, including the families that manage the largest firms in most countries in the world", La Porta, Lopez-de-Silanes, Shleifer and Vishny (2000a).
} 
propriété et de droits de vote. En France, aucune base de ce type n'existe actuellement. Par ailleurs et de manière non exhaustive :

- Les politiques de distribution sont-elles liées à la structure de l'actionnariat? Et si oui de quelle manière ? L'hypothèse de La Porta, Lopez de Silanes, Shleifer et Vishny (2000b) suivant laquelle les distributions sont plus généreuses dans les pays où les droits des minoritaires sont le plus fortement protégé, est-elle validée ?

- La rotation des dirigeants est-elle liée à la structure de l'actionnariat ?

\section{Bibliographie}

Arrow K., 1979, « The property right doctrine and demand revelation under incomplete information », in Economics and human welfare

Bagnoli M. and B.L. Lipman, 1988, "Successful takeovers without exclusion", Review of Financial Studies, Vol 1, n 1, p. 89-110.

Barclay M. and C. Holderness, 1989, Private benefits from control of public corporations, Journal of Financial Economics, 25, p. 371-395.

Barclay M.J., C.G. Holderness and J. Pontiff, 1993, "Private benefits from block ownership and discounts on closed-end funds", Journal of Financial Economics, 33, June, p. 263291.

Becht M., 1997, "Strong Blockholders, Weak Owners and the Need for European Mandatory Disclosure", The European Union (Executive Summary), (les rapports du groupe de travail "European Corporate Governance Network" sont téléchargeables àl'adresse http://www.ecgn.ulb.ac.be/ecgn/)

Becht M. and A. Roell, 1999, "Blockholdings in Europe: An International Comparison", European Economic Review, Vol. 43, No. 4-6, 1999

Berle A. and G. Means, 1932, The Modern Corporation and Private Property. MacMillan, New York.

Bhide A, 1993, The hidden cost of stock market liquidity, Journal of Financial Economics, 34, p. 31-51.

Bloch L. and E. Kremp, 1997, "Ownership and Control in France”, rapport pour la commission européenne

Brickley J.A., R.C. Lease and C.W. Smith Jr, 1988, Ownership structure and voting on antitakeover amendments, Journal of Financial Economics, Vol 20, $\mathrm{n}^{\circ} 1-2$, JanuaryMarch, p. 267-291.

Claessens S., S. Djankov and L. H.P. Lang, 2000, The separation of ownership and control in East asian corporation, Journal of Financial Economics, October, p.

Coase R., 1961, The problem of social cost, Journal of Law and Economics, 3, p 1-44.

DeAngelo H. and E. Rice, 1983, "Antitakeover Amendments and Stockholder Wealth," Journal of Financial Economics, 11, p. 329-360.

DeAngelo H. and L. DeAngelo, 1985, Managerial ownership of voting rights, Journal of Financial Economics, 14, p. 33-69.

Demirgüç-Kunt A. and V. Maksimovic, 1998, « Law, finance, and firm growth », The Journal of Finance, december

Demirgüç-Kunt A. and V. Maksimovic, 1999, « Institutions, financial markets and firm debt maturity », Journal of Financial Economics, p. 295-336

Demsetz H., 1986, "Corporate control, insider trading, and rates of return", American Economic Review, Vol 76, n 2, p. 313-316.

Easterbrook F., 1984, 'Two Agency-Cost Explanations of Dividends”, American Economic Review, 74, p 650-659. 
Easterbrook F. and D. Fischel, 1983, "Voting in Corporate Law", Journal of Law and Economics, 26, p. 395-427.

Faccio M. and L.H.P. Lang, 2000, The separation of ownership and control, an analysis of ultimate ownership in western European corporations", présenté en août à Londres au congrès de l'EFA,.

Fama E., 1980, "Agency Problems and the Theory of the Firm", Journal of Political Economy, 88, p. 288-307.

Grossman S., and O. Hart. 1988. "One-share-one-vote and the Market for Corporate Control." Journal of Financial Economics, 20, p. 175-202.

Harris M. et A. Raviv, 1988, "Corporate governance, voting rights and majority rules", Journal of Financial Economics, 20, p. 203-235.

Hart O., 1983, "The market mechanism as an incentive scheme", Bell Journal of Economics, Vol 14, n ${ }^{\circ} 2$, p. 366-382.

Hart O., 1995, Firms contracts and financial structure, Clarendon Lectures in Economics, Oxford University Press.

Holmstrom, B., and J. Tirole, 1993, "Market Liquidity and Performance Monitoring," Journal of Political Economy, 101 (August).

Husson B., B. Jacquillat B. et P. Schintowski, 1987, La valeur du droit de vote : une étude empirique des certificats d'investissement », Cahier de Recherche, HEC, $\mathrm{n}^{\circ} \mathrm{CR}$ 300/1987.

Jarrell G.A. and A.B. Poulsen, 1988, "Dual-class recapitalizations as antitakeover mechanisms, the recent evidence, Journal of Financial Economics, Vol 20, $\mathrm{n}^{\circ} 1-2$, January-March, p. 129-152.

Jensen M., 1986, "Agency Costs of Free Cash Flow, Corporate Finance and Takeovers," American Economic Review, 76 (May).

Jensen M., and W. Meckling, 1976, "Theory of the firm: Managerial behavior, agency costs, and ownership structure", Journal of Financial Economics, Vol 3, p. 305-60.

Jensen M. and R. Ruback, 1983, "The market for corporate control: the scientific evidence", Journal of Financial Economics, Vol 11, p. 5-50.

King R.G. and R. Levine, 1993, « Finance and growth: Schumpeter might be right », Quarterly Journal of Economics, 108, p. 717-738.

La Porta R. and F. Lopez-de-Silanes, 1998, « Capital market and legal institutions », WP Harvard.

La Porta R., F. Lopez-de-Silanes and A. Shleifer, 1999, « Corporate ownership around the world », The Journal of Finance, April, Vol 54, n 2, p. 471-518

La Porta R., F. Lopez-de-Silanes, A. Schleifer and R.W. Vishny, 1998, « Law and Finance », Journal of Political Economy, 106, p. 1113-1150.

La Porta R., F. Lopez-de-Silanes, A. Shleifer and R.W. Vishny, 1999, « Investor protection : origins, consequences, reform », World Bank, Financial sector discussion paper, ${ }^{\circ} 1$.

La Porta R., F. Lopez-de-Silanes, A. Shleifer and R.W. Vishny, 2000a, « Investor protection and corporate governance », Journal of Financial Economics, October, p.

La Porta, R., F. Lopez-de-Silanes, A. Shleifer, and R.W. Vishny , 2000b, "Agency Problems and Dividend Policies around the World.", Journal of Finance, forthcoming.

Lease R, J. McConnell, and W. Mikkelson, 1983, "The Market Value of Control in Publicly Traded Corporations", Journal of Financial Economics, 11,439-71.

Levine R. and S. Zervos, 1998, « Stock markets, banks and economic growth », American Economic Review, 88, p. 537-559.

Levy H., 1982, , "Economic Valuation of Voting Power of Common Stock", Journal of Finance, Vol 38, p. 79-93. 
Loderer C. et A. Jacobs, 1995, “The Nestlé crash", Journal of Financial Economics, 37, March, pp. 315-340.

Mikkelson W.H. and M.M. Partch, 1989, "Managers' voting rights and corporate control", Journal of Financial Economics, Vol 25, n 2, p. 263-290.

Modigliani F., and M. Miller, 1958, "The Cost of Capital, Corporation Finance, and the Theory of Investment.", American Economic Review, 48, p.261-97.

Morck B. Yeung, and W. Yu, 2000, « The information content of stock markets », Journal of Financial Economics, October, p. - .

Morck R., Shleifer A., and Vishny, R., 1988, Management ownership and market valuation: an empirical analysis, Journal of Financial Economics, 20, p 293-315.

Pound J., 1988, 'Proxy Contests and the Efficiency of Shareholder Oversight', Journal of Financial Economics, 20, p 237-265.

Ruback R.S., 1988, "Coercive dual-class exchange offers", Journal of Financial Economics, Vol 20, n 1-2, January-March, p. 153-173.

Rydqvist K.,1992,"Takeover Bids and the Relative Prices of Shares That Differ in Their Voting Rights", working paper, Stockholm School of Economics.

Shleifer A. and R.W. Vishny, 1997, « A survey of corporate governance », Journal of Finance, 52, June, p. 737-784.

Shleifer, A., Vishny, R., 1986. Large shareholders and corporate control, Journal of Political Economy, 94, p. 461-488.

Slovin M.B. and M.E. Sushka, 1993, "Ownership concentration, corporate control activity, and firm value: evidence from the death of inside blockholders", Journal of Finance, Vol 48, n 4, p. 1293-1321.

Stulz, R, 1988, Managerial control of voting rights: Financing policies and the market for corporate control, Journal of Financial Economics 20, 25-54.

Tnani S., 1997, Les incidences des modifications de la structure de l'actionnariat sur la richesse des actionnaires, études empiriques sur le marché français, Thèse pour le Doctorat de Sciences de Gestion, Université Paris-Dauphine.

Wurgler J., 2000, «Financial markets and the allocation of capital», Journal of Financial Economics, October

Zingales L. 1994. "The Value of the Voting Right: a Study of the Milan Stock Exchange.", The Review of Financial Studies, 7, 125-148. 